\title{
Singularities of Affine Schubert Varieties ${ }^{\star}$
}

\author{
Jochen KUTTLER ${ }^{\dagger}$ and Venkatramani LAKSHMIBAI ${ }^{\ddagger}$ \\ $\dagger$ Department of Mathematical \& Statistical Sciences, University of Alberta, Edmonton, Canada \\ E-mail: jochen.kuttler@ualberta.ca \\ $¥$ Department of Mathematics, Northeastern University, Boston, USA \\ E-mail: lakshmibai@neu.edu
}

Received September 11, 2008, in final form April 03, 2009; Published online April 18, 2009

doi:10.3842/SIGMA.2009.048

\begin{abstract}
This paper studies the singularities of affine Schubert varieties in the affine Grassmannian (of type $\mathrm{A}_{\ell}^{(1)}$ ). For two classes of affine Schubert varieties, we determine the singular loci; and for one class, we also determine explicitly the tangent spaces at singular points. For a general affine Schubert variety, we give partial results on the singular locus.
\end{abstract}

Key words: Schubert varieties; affine Grassmannian; loop Grassmannian

2000 Mathematics Subject Classification: 14M15; 14L35

\section{Introduction}

Schubert varieties are important objects in the theory of algebraic groups, representation theory, and combinatorics. The determination of their singularities is a classical problem and has been studied by many authors. Very conclusive results are available for groups of type $A$ (see e.g. $[16,15,4,7,11,22]$; and for arbitrary types $[3,12,6])$. In this note we investigate singularity properties of the natural generalization of Schubert varieties to affine Schubert varieties. In the affine setting, the question has not been settled yet, although, there are certainly results available (in particular, [12] applies as well). Recently, affine Schubert varieties (in all types) have been studied by several authors (cf. [2, 8, 10, 21]). While in [8, 10, 21], the authors study the singularities of $\mathcal{P}$-stable affine Schubert varieties (see Section 6.1), in [2], the authors classify the smooth and rationally smooth Schubert varieties.

The most classical Schubert varieties are the Schubert varieties in Grassmannians, and the first generalization is therefore to the affine Schubert varieties in the affine Grassmannian of type $A^{(1)}$. So let us fix an algebraically closed field $K$ of characteristic zero, and denote by $A=K[[t]]$ the ring of formal power series with quotient field $F=K((t))$, the ring of formal Laurent series. Then $\mathrm{SL}_{n}(A)$ and $\mathrm{SL}_{n}(F)$ both are the $K$-points of ind-varieties over $K$, denoted by $\mathcal{P}$ and $\mathcal{G}$, and $\mathcal{P} \subset \mathcal{G}$. The affine Grassmannian is then the quotient ind-variety $\mathcal{G} / \mathcal{P}$. Mimicking the classical situation the affine Schubert varieties are the $\mathcal{B}$-orbit closures in $\mathcal{G} / \mathcal{P}$, where $\mathcal{B} \subset \mathcal{P}$ is the subgroup of elements where the (strictly) upper triangular entries are divisible by $t$; more formally $\mathcal{B}=\mathrm{ev}^{-1}(B)$, where $B \subset \mathrm{SL}_{n}(K)$ is the Borel subgroup of lower triangular matrices and ev: $\mathcal{P} \rightarrow \mathrm{SL}_{n}(K)$ is the evaluation homomorphism sending $\left[g_{i j}(t)\right]$ to $\left[g_{i j}(0)\right]$. Let $T$ be the maximal torus consisting of diagonal matrices in $\mathrm{SL}_{n}(K) \subset \mathcal{P}$, and let $S=K^{*}$ be the one-dimensional torus in $\operatorname{Aut}(\mathcal{G})$ coming from the action of $S$ on $F$ by rotating the loops, i.e. $s \in S$ sends $g(t)$ to $g(s t)$. As the $S$ and $T$-actions commute, putting $\widehat{T}=T \times S$ we obtain an $n$-dimensional torus acting on $\mathcal{G}, \mathcal{P}$ and $\mathcal{B}$, and therefore on any affine Schubert variety. Each $\mathcal{B}$-orbit contains a unique $\widehat{T}$-fixed point; the $\widehat{T}$-fixed points in $\mathcal{G} / \mathcal{P}$ are parameterized by $\widehat{W}^{\mathcal{P}}$,

\footnotetext{
${ }^{\star}$ This paper is a contribution to the Special Issue on Kac-Moody Algebras and Applications. The full collection is available at http://www.emis.de/journals/SIGMA/Kac-Moody_algebras.html
} 
a set of representatives of $\widehat{W} / W, \widehat{W}$ (resp. $W$ ) being the affine Weyl group (resp. the Weyl group) of type $\mathrm{A}_{n-1}^{(1)}$ (resp. $\mathrm{A}_{n-1}$ ). In fact, $\widehat{W}^{\mathcal{P}}$ has a natural identification with $\mathbb{Z}^{n-1}$ embedded in $\mathbb{Z}^{n}$ as the sublattice consisting of points in $\mathbb{Z}^{n}$ with coordinate sum being equal to zero. Let $\geq$ denote the partial order on $\widehat{W}^{\mathcal{P}}$ induced by the partial order on (the Coxeter group) $\widehat{W}$ (with respect to the set of simple roots associated to $\mathcal{B})$. For $w \in \widehat{W}^{\mathcal{P}}$, let

$$
X(w):=\bigcup_{\left\{v \in \widehat{W}^{\mathcal{P}}, v \leq w\right\}} \mathcal{B} v
$$

be the affine Schubert variety in $\mathcal{G} / \mathcal{P}$ associated to $w$. (Thus for $v, w \in \widehat{W}^{\mathcal{P}}$, we have, $v \leq w$ if and only if $X(v) \subseteq X(w))$.

In this paper, for studying the affine Grassmannian and the affine Schubert varieties, we make use of a canonical embedding of affine Grassmannian into the infinite Grassmannian $\operatorname{Gr}(\infty)$ over $K$ (cf. Section 2). We briefly explain below our approach.

$\operatorname{Gr}(\infty)$ being the inductive limit of all finite dimensional Grassmannians, we obtain a canonical identification of an affine Schubert variety $X(w)$ as a closed subvariety of a suitable Grassmannian $G(d, V)$ (the set of $d$-planes in the vector space $V$ ), in fact, as a closed subvariety of a suitable classical Schubert variety in $G(d, V)$. Further, as a subset of $\operatorname{Gr}(\infty)$, we get an identification of $\mathcal{G} / \mathcal{P}$ with the set of $A$-lattices in $F^{n}$ (i.e., free $A$-submodules of $F^{n}$ of rank $n$ ). For instance, the element $\left(c_{1}, \ldots, c_{n}\right) \in \widehat{W}^{\mathcal{P}}$ corresponds to the $A$-span of $\left\{t^{c_{1}} e_{1}, \ldots, t^{c_{n}} e_{n}\right\}$ (here, $\left\{e_{1}, \ldots, e_{n}\right\}$ is the standard $F$-basis for $\left.F^{n}\right)$. Given $w=\left(c_{1}, \ldots, c_{n}\right) \in \widehat{W}^{\mathcal{P}}$, there exists an $s>0$ such that $w \leq w_{0}^{s}$ with $w_{0}^{s}:=(-s(n-1), s, s, \ldots, s)\left(\in \widehat{W}^{\mathcal{P}}\right)$, and hence any $X(w)$ may be thought of as a subvariety of $X\left(w_{0}^{s}\right)$, for a suitable $s$. We have an identification (cf. Section 2.4)

$$
X\left(w_{0}^{s}\right)=\left\{A \text {-lattice } L \mid t^{-s(n-1)} L_{0} \supset L \supset t^{s} L_{0}, \text { and } \operatorname{dim}_{K}\left(L / t^{s} L_{0}\right)=s n\right\},
$$

$L_{0}$ being the standard lattice (namely, the $A$-span of $\left\{e_{1}, \ldots, e_{n}\right\}$ ). Setting $V:=t^{-s(n-1)} L_{0} / t^{s} L_{0}$, we have $\operatorname{dim} V=s n^{2}$; further, the map $f_{s}: X\left(w_{0}^{s}\right) \rightarrow G(d, V), L \mapsto L / t^{s} L_{0}$ (where $d:=s n$ ) identifies $X\left(w_{0}^{s}\right)$ as a closed subvariety of $G(d, V)$. Denoting $u:=1+t$, the unipotent endomorphism of $V, v \mapsto v+t v$, we have that $u$ induces an automorphism of $G(d, V)$ and $f_{s}$ identifies $X\left(w_{0}^{s}\right)$ with $G(d, V)^{u}$ (the fixed point set of $u$ with the reduced scheme structure) (cf. Proposition 2.1). Moreover, for each affine Schubert variety $X(w) \subseteq X\left(w_{0}^{s}\right)$, we have that $w \in G(d, V)$ is a $\mathfrak{T}$-fixed point (for a suitable maximal torus $\mathfrak{T}$ of $\mathrm{GL}(V)$ ), giving rise to a classical Schubert variety $Y(w)$ (with respect to a suitable Borel subgroup $\mathfrak{B}$ ) which is $u$-stable, and we have an identification: $X(w)=Y(w)^{u}$. Thus we exploit this situation to deduce properties for affine Schubert varieties. In particular, for two classes of affine Schubert varieties contained in $X\left(w_{0}^{S}\right)$, we determine explicitly the singular loci; further, for one of the two classes, we also determine the tangent spaces at singular points. In order to describe our results, given $w=\left(c_{1}, c_{2}, \ldots, c_{n}\right)\left(\in \widehat{W}^{\mathcal{P}} \subset \mathbb{Z}^{n}\right)$, such that $X(w) \subseteq X\left(w_{0}^{s}\right)(\subset G(d, V))$, we define $L(w):=\left(l_{1}, l_{2}, \ldots, l_{2 n}\right)$, where

$$
l_{i}= \begin{cases}s-c_{i}, & \text { if } i \leq n, \\ s-c_{i-n}+1, & \text { if } i>n .\end{cases}
$$

The first class of affine Schubert varieties that we consider consists of the $\mathcal{P}$-stable affine Schubert varieties. Note that the $\mathcal{P}$-stable affine Schubert varieties $X(w)$ 's contained in $X\left(w_{0}^{s}\right)$ may be characterized by the corresponding $L(w)$ 's: $X(w)$ is $\mathcal{P}$-stable if and only if $l_{1} \geq l_{2} \geq$ $\cdots \geq l_{n}$. The Schubert variety $X\left(w_{0}^{s}\right)$ is an example of a $\mathcal{P}$-stable Schubert variety, and an important at that, in view of its relationship (cf. [19]) with nilpotent orbit closures in $\operatorname{Lie}(G)$ (for the adjoint action of $G$ on $\operatorname{Lie}(G) G$ being $\left.\mathrm{GL}_{n}(K)\right)$. To be very precise, for $s=1, X\left(w_{0}^{s}\right)$ contains the variety of nilpotent matrices as an open subset; moreover, we have a bijection 
between $\{$ nilpotent orbit closures $\}$ and $\left\{\mathcal{P}\right.$-stable affine Schubert subvarieties of $\left.X\left(w_{0}^{1}\right)\right\}$ - by Lusztig's isomorphism (cf. [19]), a nilpotent orbit closure gets identified with an open subset (namely, the "opposite cell") of an unique Schubert subvariety of $X\left(w_{0}^{1}\right)$. For a $\mathcal{P}$-stable affine Schubert variety $X$, we determine Sing $X$, the singular locus of $X$; of course, this result was first proved by Evens and Mircović (cf. [8]). We give yet another proof of their result, which is that the regular locus of a $\mathcal{P}$-stable Schubert variety is precisely the open $\mathcal{P}$-orbit. We also determine explicitly (cf. Corollary 4.9, Theorems 4.16, 6.9) $T_{x} X\left(w_{0}^{s}\right)$, the tangent space to $X\left(w_{0}^{s}\right)$ at $x \leq w_{0}^{s}$; our description is in terms of $T_{x} Y\left(w_{0}^{s}\right)$, for any $s>0$.

The second class of affine Schubert varieties consists of $X(w)$ 's such that $L(w)$ admits two indices $i \leq n, i \leq j<i+n$, such that for $k=i, \ldots, i+n-1, k \neq j$, we have $l_{k}$ is independent of $k$, and less than or equal $l_{j}$ (cf. Section 6); we say that $w$ consists of one string. Note that $w_{0}^{s}$ consists of one string. For these $X(w)$ 's, we show (cf. Theorem 6.9):

Theorem A. Let $w$ consist of one string. Then $T_{x} X(w)=T_{x} X\left(w_{0}^{s}\right) \cap T_{x} Y(w)$.

We also prove the rational smoothness of $X\left(w_{0}^{s}\right)$ and certain of the Schubert varieties of the "one-string" type (cf. Theorem 6.12 and its corollary), which is also obtained in [2].

The realization of the affine Schubert variety $X(w)$ as a closed subvariety of the classical Schubert variety $Y(w)(\subset G(d, V))$ enables us to construct certain singularities as explained below. We say $P=(i, j)(i<j)$ is an imaginary pattern in $L(w)$ if $l_{i}>l_{j}+1$. We may assume $i \leq n$.

Theorem B. Let $P=(i, j)$ be an imaginary pattern in $w$ and let $w_{P}$ be defined by $L\left(w_{P}\right)_{i}=$ $l_{i}-1, L\left(w_{P}\right)_{j}=l_{j}+1$, and $L\left(w_{P}\right)_{k}=L(w)_{k}, k \neq i, j$. Then $w_{P}$ is a singular point of $X(w)$.

It turns out that Theorem B is enough to describe the maximal singularities of the two classes of Schubert varieties described above.

The reason why $w_{P}$ is singular is simply that $T_{w_{P}}(X(w))$ contains a tangent line whose $\widehat{T}$-weight is an imaginary root. As these are never tangent to $\widehat{T}$-stable curves, $w_{P}$ has to be singular. Of course, another possible reason for singularity is that there may be too many of such curves. It is not hard to construct points in most Schubert varieties where this is the case: Let $P: i<g<j<k \leq 2 n$ be a sequence with $i \leq n$, such that $j<i+n, k<g+n$, and $l_{i} \geq l_{j}>l_{g} \geq l_{k}$. We call $P$ a real pattern of the first kind. Define $w_{P}$ by putting $\left(l_{g}, l_{k}, l_{i}, l_{j}\right)$ in $L(w)$ at positions $(i, g, j, k)$ (see Section 5). Similarly, let $Q: i<j<g<k \leq 2 n$ be a sequence of integers such that $i \leq n, g<i+n, k<j+n$, and $l_{j}>l_{i} \geq l_{k}>l_{g}$. We refer to $Q$ as a real pattern of the second kind. Define $w_{Q}$ by putting $\left(l_{g}, l_{k}, l_{i}, l_{j}\right)$ in $L(w)$ at positions $(i, j, g, k)$.

Theorem C. If $w$ admits a real pattern $P$ of any kind then $w_{P}$ is a singular point in $X(w)$.

As in the classical setting, the geometric explanation why $w_{P}$ is singular is that the dimension of $T_{x}(X(w))$ is too big due to the presence of too many $\widehat{T}$-invariant curves each of whom contributes a line in $T_{x}(X(w))$.

Remark 1.1. We observe that the relative order of the lengths $l_{i}, l_{j}, l_{g}, l_{k}$ in both of the real patterns is almost the same as for the Type I and II patterns for classical Schubert varieties (cf. [15]). The difference is that we allow non-strict inequalities at some places. Also, if $P$ is such a pattern the relative order of the lengths in $w_{P}$ is the same as in the singularity constructed from the pattern in the classical setting.

Of course this begs the question whether the results of the classical setting could be applied directly to show that $w_{P}$ is singular. While in some examples this seems indeed possible we haven't been able so far to make this precise except for "obvious" cases. Hopefully we will be able to address this question more satisfactorily in some future work. We give some indication in Remark 5.8. 
The same can be said for the more general question, whether we can actually formulate these pattern in terms of the Weyl group elements and relate it to work of Billey-Braden [1] or Billey-Postnikov [3] in the finite case.

Of course, this raises the question whether all (maximal) singularities arise in this fashion. So far we haven't been able to answer this question. However, in many examples it is true, if one allows two degenerated cases of real patterns as well (as discussed in Section 5).

The paper is organized as follows: In Section 2, we establish the basic notation and conventions used throughout the text, and we will collect some elementary results which will help further on. Section 3 introduces the main combinatorial tools as well as the notion of "small" reflections and the language of up-/down-exchanges, which serve as a tool in describing the singularities later. In Section 4 we investigate the relation between a Schubert variety $X(w)$ and the classical Schubert variety $Y(w)$ which contains it, as far as tangent spaces are concerned, and we introduce imaginary and real tangents. Section 5 again returns to combinatorics, precisely defining the various patterns in $w$ which give rise to singularities, and proving Theorems A and B. The final Section 6 then applies these results to two classes of Schubert varieties, those consisting of "one string", and those that are $\mathcal{P}$-stable.

\section{Preliminaries}

As explained in the Introduction, for the study of the affine Schubert varieties in $\mathcal{G} / \mathcal{P}$, we make use of a canonical embedding (as an Ind-subvariety) of $\mathcal{G} / \mathcal{P}$ into $\operatorname{Gr}(\infty)$, the infinite Grassmannian. We shall now describe this embedding. For details, we refer the readers to [13] and [20].

\subsection{The affine and infinite Grassmannians}

We will keep the notation already established in the Introduction. Consider the $K$-vector space $K^{\infty}$ of $K$-valued functions on $\mathbb{Z}$ that vanish on "very negative" values, i.e. $K^{\infty}=\{f: \mathbb{Z} \rightarrow$ $K \mid f(i)=0 ; i \ll 0\}$. For each $i \in \mathbb{Z}$, there exists a canonical element $e_{i} \in K^{\infty}$ defined by $e_{i}(j)=\delta_{i j}$. Then every $f \in K^{\infty}$ may be written formally as $f=\sum_{i \in \mathbb{Z}} f_{i} e_{i}$ where $f_{i}=f(i)$. Let $E_{r}=\left\{f \in K^{\infty} \mid f(i)=0, \forall i<r\right\}$ be the "span" of $e_{r}, e_{r+1}, \ldots$ The infinite Grassmannian $\operatorname{Gr}(\infty)$ over $K$ is by definition the Ind-scheme obtained as the direct limit of usual Grassmannians as outlined below. Its $K$-valued points are given by linear subspaces $E$ of $K^{\infty}$, such that for some $r>0, E_{r} \subset E$ and $E / E_{r}$ is finite-dimensional. Obviously, for such an $E$ we may increase $r$ if necessary so that $E_{r+1} \subset E \subset E_{-r}$. As both $E$ and $E_{1}$ contain $E_{r+1}$, we have

$$
\operatorname{dim} E / E_{r+1}=r+\operatorname{dim} E /\left(E_{1} \cap E\right)-\operatorname{dim} E_{1} /\left(E_{1} \cap E\right) .
$$

We set $\operatorname{vdim}(E):=\operatorname{dim} E_{1} /\left(E_{1} \cap E\right)-\operatorname{dim} E /\left(E_{1} \cap E\right)$. For any $K$-vector space $V$ and any positive integer $d$, let $G(d, V)$ denote the Grassmannian of $d$-planes in $V$. Then, for $s$ sufficiently large, we have that $E$ is naturally an element of $G\left(r-\operatorname{vdim}(E), E_{-s} / E_{r+1}\right)$. The Grassmannians $\{G(r-i$, $\left.\left.\left.E_{-s} / E_{r+1}\right)\right)_{s>0, r \geq i}\right\}$ ( $i$ being fixed) form a direct system of varieties, with $G\left(r-i, E_{-s} / E_{r+1}\right)$ and $G\left(r^{\prime}-i, E_{-s^{\prime}} / E_{r^{\prime}+1}\right)$ both mapping naturally to $G\left(r+r^{\prime}-i, E_{-s-s^{\prime}} / E_{r+r^{\prime}+1}\right)$. Its limit Ind-variety is denoted $\operatorname{Gr}(\infty)_{i}$, and it parameterizes all $E$ with $\operatorname{vdim}(E)=i$. Thus, $\operatorname{Gr}(\infty)=$ $\bigcup_{i \in \mathbb{Z}} \operatorname{Gr}(\infty)_{i}$ carries a natural Ind-variety structure.

Let $\mathfrak{X}$ be the set of all $A$-lattices in $F^{n}$. Then $\mathfrak{X}$ is naturally an algebraic subset of the set of $K$-valued points of $\operatorname{Gr}(\infty)$ and therefore carries a structure as an Ind-scheme. Indeed, let $F^{n} \rightarrow K^{\infty}$ be the isomorphism that sends $t^{i} v_{j}$ to $e_{j+i n},\left\{v_{j}, 1 \leq j \leq n\right\}$ being a $F$-basis of $F^{n}$. Since for every $A$-lattice $L \subset F^{n}, F^{n} / L$ is a torsion module, $L$ contains $t^{r} A^{n}$ for a suitably large $r>0$. On the other hand, if $\left\{u_{i}, 1 \leq j \leq n\right\}$ is an $A$-basis for $L$, and ord $u_{i} \geq N, \forall i$ for some 
$N \in \mathbb{Z}$, then $L \subset t^{N} A^{n}$. Thus the image of $L$ in $K^{\infty}$ defines naturally an element of $\operatorname{Gr}(\infty)$; further, we have,

$$
\mathfrak{X}=\{E \in \operatorname{Gr}(\infty) \mid t E \subseteq E\} .
$$

Here, via the identification of $F^{n}$ with $K^{\infty}, t$ acts on $K^{\infty}$ as $t e_{i}=e_{i+n}$; in the sequel, we shall denote the map $K^{\infty} \rightarrow K^{\infty}, e_{i} \mapsto e_{i+n}$, by $\tau$. Notice that $\tau$ defines a nilpotent map on each $E_{-r} / E_{r+1}$, while the left-multiplication by $t$ defines an automorphism of $\operatorname{Gr}(\infty)$.

To describe the Ind-variety structure on $\mathcal{G} / \mathcal{P}$, we consider the natural transitive action of $\mathrm{GL}_{n}(F)$ on $\mathfrak{X}$; observe that $\mathrm{GL}_{n}(A)$ is the stabilizer of the standard lattice $A^{n} \subset F^{n}$. Hence, we get an identification $\mathrm{GL}_{n}(F) / \mathrm{GL}_{n}(A) \cong \mathfrak{X}(\subset \operatorname{Gr}(\infty))$. Now $\mathcal{G}$ acts naturally on $\operatorname{Gr}(\infty)$ and on $\mathfrak{X}$, and it is easily seen that $\mathfrak{X} \cap \operatorname{Gr}(\infty)_{0}$ is exactly one $\mathcal{G}$-orbit with $\mathcal{P}$ as the stabilizer of the standard lattice. Thus we obtain an embedding $\mathcal{G} / \mathcal{P} \hookrightarrow \operatorname{Gr}(\infty)_{0}$ identifying $\mathcal{G} / \mathcal{P}$ as an Ind-subvariety of $\operatorname{Gr}(\infty)_{0}$.

\subsection{The Weyl group}

Let $G=\mathrm{SL}_{n}(K)$ with Lie algebra $\mathfrak{g}$. Let $\Phi$ be the root system of $(G, T)$, and let $W=\mathfrak{S}_{n}$ be the associated Weyl group. The associated affine root system $\widehat{\Phi}$ then is by definition the set of roots of $\widehat{T}$ in $\mathfrak{g} \otimes_{K} K\left[t, t^{-1}\right]$. It may be identified with $\Phi \times \mathbb{Z} \cup\{0\} \times \mathbb{Z}$, and we write $\delta$ for $(0,1) \in \widehat{\Phi}$. The elements of $\mathbb{Z} \delta$ are called imaginary roots, and all other elements are called real roots. For $\hat{\alpha}=\alpha+h \delta$ with $\alpha \in \Phi$ we put $\Re(\hat{\alpha})=\alpha$. A root $\hat{\alpha}=\alpha+h \delta \in \widehat{\Phi}$ is positive if $h>0$, or $h=0$ and $\alpha$ is positive (in the usual sense with respect to $\Phi$ ); otherwise, $\hat{\alpha}$ is called negative.

Let $\mathfrak{S}_{\infty}$ be the group of permutations of $\mathbb{Z}$, and $\tau$ the element in $\mathfrak{S}_{\infty}: \tau(i)=i+n, i \in \mathbb{Z}$. Let $\widetilde{W}=\left\{\sigma \in \mathfrak{S}_{\infty} \mid \tau \sigma=\sigma \tau\right\}$. Clearly $\widetilde{W}=W \rtimes \mathbb{Z}^{n}$, where $W$ embeds naturally into $\mathfrak{S}_{\infty}$, acting on the intervals $[1+k n,(k+1) n]$, and $\mathbb{Z}^{n}$ embeds as $c=\left(c_{1}, \ldots, c_{n}\right)$ maps to $\tau^{c}$ with $\tau^{c}(i+k n):=i+\left(k+c_{i}\right) n$. The Weyl group $\widehat{W}$ of $(\mathcal{G}, \widehat{T})$ may be naturally identified with a subgroup of $\widetilde{W}$.

$\widehat{W} \subset \widetilde{W}$ is given as the set of those $(w, c) \in \widetilde{W}$ with $\sum_{i} c_{i}=0$. It is generated by reflections $s_{\hat{\alpha}}$ associated to the real roots $\hat{\alpha} \in \Phi$ : For a root $\alpha=(i j) \in \Phi$ write $c_{\alpha}=e_{j}-e_{i} \in \mathbb{Z}^{n}$ where $\left\{e_{k}\right\}$ denotes the standard basis of $\mathbb{Z}^{n}$. If $\hat{\alpha}=\alpha+h \delta \in \widehat{\Phi}$ with $\alpha$ being a positive root in $\Phi$, then $s_{\hat{\alpha}}=\left(s_{\alpha}, h c_{\alpha}\right) \in \widetilde{W}$, where $s_{\alpha} \in W$ is the permutation associated to $\alpha$. If $\alpha=(i j)$ with $i>j$, then

$$
s_{\hat{\alpha}}(q)= \begin{cases}j+(k-h) n, & \text { if } q=i+k n, \\ i+(k+h) n, & \text { if } q=j+k n, \\ q, & \text { if } q \neq \equiv i \text { or } j(\bmod n) .\end{cases}
$$

Further, $s_{-\hat{\alpha}}$ is then defined as $s_{\hat{\alpha}}$.

\subsection{Schubert varieties}

As mentioned in the introduction, each $\mathcal{B}$-orbit on $\mathcal{G} / \mathcal{P}$ contains a unique $\widehat{T}$-fixed point. In fact, these fixed points form one orbit under the natural action of $\widehat{W}$. These are best described in the language of lattices. Clearly an $A$-lattice $L \subset F^{n}=K^{\infty}$ is normalized by $\widehat{T}$ if and only if it has a basis of the form $e_{i_{1}}, e_{i_{2}}, \ldots, e_{i_{n}}$; it is clear that as an element of $\operatorname{Gr}(\infty), L$ has the form $L=V_{0} \oplus E_{r}$ for some $r>0$, and some subspace $V_{0}$ spanned by a subset of the natural basis of $E_{-r} / E_{r}$. Therefore $L$ is uniquely determined by the ascending sequence $w(L)$ of integers, describing which $e_{i}$ lie in $L: w(L)=\left(w_{1}, w_{2}, \ldots\right)$, and $w_{i}$ occurs if and only if $e_{w_{i}} \in L$. Notice that eventually $w(L)$ agrees with the natural sequence since $E_{r} \subset L$ for some $r$. One checks easily that $L=\tau^{c} E_{1}$ for a suitable $c$, and in fact

$$
\mathbb{Z}^{n} \rightarrow \mathfrak{X}^{\widehat{T}}, \quad c \mapsto \tau^{c} E_{1}
$$


is a bijection. Notice that $\operatorname{vdim}\left(\tau^{c} E_{1}\right)=\sum_{i} c_{i}$. Further, as seen above, $\mathfrak{X} \cap \operatorname{Gr}(\infty)_{0}=\mathcal{G} / \mathcal{P}$, and the $\widehat{T}$-fixed points therein are given by the image of $\mathbb{Z}^{n-1}$ under the map (1). We thus obtain a notion of Schubert variety in all of $\mathfrak{X}$ (these are of course the Schubert varieties for $\left.\mathrm{GL}_{n}(F) / \mathrm{GL}_{n}(A)\right)$. Recall that $t$ acts as an automorphism of $\operatorname{Gr}(\infty)$ and $\mathfrak{X}$, which commutes with the action of $\mathcal{G}$. Clearly $t\left(\operatorname{Gr}(\infty)_{i}\right)=\operatorname{Gr}(\infty)_{i+n}$ for all $i$, and under this map $\mathcal{B}$-orbit closures are sent to $\mathcal{B}$-orbit closures. For any $w \in \mathbb{Z}^{n}$ we put $X(w)=\overline{\mathcal{B} w} \subset \mathfrak{X}$. Here we are only interested in those $X(w)$ which lie in $\operatorname{Gr}(\infty)_{0}$.

\subsection{The Schubert variety $X\left(w_{0}^{s}\right)$}

For $s>0$, let $w_{0}^{s}=\tau^{(-s(n-1), s, \ldots, s)}$. Clearly, the lattice $L_{w_{0}^{s}}\left(=\tau^{(-s(n-1), s, \ldots, s)} E_{1}\right)$ has the property $t^{-s(n-1)} L_{0} \supset L_{w_{0}^{s}} \supset t^{s} L_{0}$ and $\operatorname{dim}_{K}\left(L / t^{s} L_{0}\right)=s n$ (here, $L_{0}$ is the standard $A$ lattice, namely, the $A$-span of $\left.\left\{e_{1}, \ldots, e_{n}\right\}\right)$. Further, for $w=\tau^{\left(c_{1}, \ldots, c_{n}\right)} \in \widehat{W}^{\mathcal{P}}$, it is easily seen that $w \leq w_{0}^{s}$ if and only if the lattice $L_{w}\left(=\tau^{\left(c_{1}, \ldots, c_{n}\right)} E_{1}\right)$ has the property $t^{-s(n-1)} L_{0} \supset L \supset$ $t^{s} L_{0}$ and $\operatorname{dim}_{K}\left(L / t^{s} L_{0}\right)=s n$. Thus, we get an identification:

$$
X\left(w_{0}^{s}\right)=\left\{A \text {-lattice } L \mid t^{-s(n-1)} L_{0} \supset L \supset t^{s} L_{0}, \text { and } \operatorname{dim}_{K}\left(L / t^{s} L_{0}\right)=s n\right\} .
$$

It is well known that $\mathcal{G} / \mathcal{P}=\lim _{\longrightarrow} X\left(w_{0}^{s}\right)$. We therefore restrict our attention to the discussion of $X\left(w_{0}^{s}\right)$. Mainly for notational convenience we replace $X\left(w_{0}^{s}\right)$ by $t^{s(n-1)} X\left(w_{0}^{s}\right)=: X\left(w^{s}\right)$ where $w^{s}=\tau^{(0, s n, \ldots, s n)}$. Let $d=s n$ (which we will keep throughout the text). As an element of $\operatorname{Gr}(\infty), w_{0}^{s}$ contains $E_{s n+1}$ and is contained in $E_{1-s(n-1) n)}$. Hence $X\left(w_{0}^{s}\right)$ embeds into $G\left(d, E_{1-d(n-1)} / E_{d+1}\right)$. Consequently $X\left(w^{s}\right)$ may be thought of as a subset of

$$
t^{d-s} G\left(d, E_{1-d(n-1)} / E_{d+1}\right)=G\left(d, E_{1} / E_{d n+1}\right) .
$$

We will denote $E_{1} / E_{d n+1}$ by $V_{s}$ or simply $V$.

Let $u:=1+t \in \mathrm{GL}(V), u(v)=v+t v, v \in V$; then $u$ is unipotent and clearly $X\left(w^{s}\right) \subseteq$ $G(d, V)^{u}$. We shall now show that this inclusion is in fact an equality.

Proposition 2.1. With notations as above, we have, $X\left(w^{s}\right)=G(d, V)^{u}$ (the fixed point set of $u$ with the reduced scheme structure).

Before we can prove this proposition we need to introduce some notation also used throughout the rest of the paper. Choosing the basis on $V$ given by $e_{1}, e_{2}, \ldots, e_{s n^{2}} \in E_{1}$, let $\mathfrak{T} \subset \operatorname{GL}(V)$ be the induced diagonal torus and let $\mathfrak{B}$ be the Borel subgroup of lower triangular matrices. Notice that $\mathcal{P}$ acts on $V$ by means of a representation $\mathcal{P} \rightarrow \operatorname{GL}(V)$, and the image of $\mathcal{B}$ is contained in $\mathfrak{B}$. Similarly, $\widehat{T}$ acts on $V$ and injects into $\mathfrak{T}$. By construction all the $\widehat{T}$-fixed points in $X\left(w^{s}\right)$ are actually $\mathfrak{T}$-fixed points. The $\mathfrak{T}$-fixed points in $G(d, V)$ are the $d$-spans $K e_{i_{1}}+K e_{i_{2}}+\cdots+K e_{i_{d}}$, where $1 \leq i_{1}<i_{2}<\cdots<i_{d} \leq d n$. We shall denote such $d$-tuples by $I_{d}$ or just $I$. An element $x=\left(x_{1}<x_{2}<\cdots<x_{d}\right) \in I$ determines (uniquely) a point of $\operatorname{Gr}(\infty)$, namely, the subspace of $K^{\infty}$ given by

$$
K e_{x_{1}}+\cdots+K e_{x_{d}}+\sum_{j>s n^{2}} K e_{j}
$$

we shall denote it by $E_{|x|}$ where

$$
|x|=x \cup \mathbb{Z}_{>s n^{2}}
$$

Note that $E_{|x|}$ contains $E_{s n^{2}+1}$. Now $E_{|x|}$ is in $\mathfrak{X}$ if and only if the underlying space is $t$-stable if and only if $x$ is a $u$-fixed point if and only if for all $y \in|x|, y+n \in|x|$ if and only if $x_{i}+n \in|x|$. 
Let $I^{u}$ denote the set of all $x$ with this property. We will identify an element $x \in I^{u}$ with its counterpart $\tau^{c} \in \widehat{W}$.

Recall the Bruhat-Chevalley order $\succeq$ on $I$ with respect to $\mathfrak{B}$ : it is defined as $v \succeq w$ if $\overline{\mathfrak{B} v} \supseteq \mathfrak{B} w$. A similarly defined order (now with respect to $\mathcal{B}$ ) exists on the set of affine Schubert varieties. For $I$ we also have the combinatorial partial order given by $v \geq w$ if and only if $v_{i} \leq w_{i}$ for all $i=1,2, \ldots, d$. A fundamental result in the theory of Schubert varieties is the fact that the two orderings on $I$ coincide, and moreover it is compatible with the order on the Weyl group of $\mathrm{GL}(V)$ given by the simple generators.

Therefore we obtain two orderings on $I^{u}$, one stemming from the Bruhat-Chevalley order on $\mathfrak{B}$-Schubert varieties, and one for the affine Schubert varieties parameterized by $I^{u}$. It is clear that the inherited order is a priori weaker than the Bruhat-Chevalley order (with respect to $\mathcal{B}$ ) on $I^{u}$. However, if $v \geq w$ in $I^{u}$, then also $X(v) \supset X(w)$ which can be shown combinatorially, or geometrically by observing that for $v \in I^{u}, \mathcal{B} v$ is dense in $\overline{\mathfrak{B} v}^{u}$.

Proof of Proposition 2.1. As mentioned above, the Bruhat-Chevalley partial order on $I^{u}$ can be described combinatorially, and hence the partial order on Schubert varieties in $X\left(w_{0}^{s}\right)$ can be described combinatorially. The $d$-tuple in $I^{u}$ (namely, $(1, n+1,2 n+1, \ldots,(d-1) n+1)$ ) representing $w^{s}$ is the largest in $I^{u}$. Hence it follows that, as sets, $X\left(w^{s}\right)=G(d, V)^{u}$.

We will now simply write $\leq$ to denote the partial order on $I$ and $I^{u}$. Note that as a consequence of the fact that the partial orders on $I^{u}$ coincide, for every $w \leq w^{s} \in I^{u}$, we have $X(w)=Y(w)^{u}$, where for every $w \in I, Y(w)$ denotes the $\mathfrak{B}$-Schubert variety $\overline{\mathfrak{B} w}$.

The Schubert varieties in $\mathcal{G} / \mathcal{P}$ share many properties with their classical counterparts, both geometrically, and combinatorially.

We finish this section by a well known but nevertheless important lemma, whose proof in its current form was pointed out to us by one of the referees.

Lemma 2.2. The action of $\widehat{T}$ (resp. $\mathfrak{T})$ on $G(d, V)$ is locally linearizable, that is, $G(d, V)$ is covered by open affine $\widehat{T}$-stable (resp. $\mathfrak{T}$-stable) neighborhoods. Consequently the same applies for every closed $\widehat{T}$-stable (resp. $\mathfrak{T}$-stable) subvariety.

Proof. The open $\mathfrak{B}$-orbit in $G(d, V)$ is both, affine and $\widehat{T}$ - (resp. $\mathfrak{T}$-) stable. Its $\mathfrak{W}$-translates cover all of $G(d, V)$.

\section{Reflections and combinatorics}

Let $d, V, Y(w)$ etc., be as in the previous section. Let $\mathfrak{W}$ be the Weyl group of the pair $(\mathrm{GL}(V), \mathfrak{T})$, and let $\mathcal{R}$ denote the set of reflections in $\mathfrak{W}$. Let $x \in Y(w)$ be a $\mathfrak{T}$-fixed point. Set

$$
S_{x}(w)=\{r \in \mathcal{R} \mid x \neq r x(\text { in } G(d, V)) \text { and } r x \leq w\} .
$$

Then we have (cf. [16]) that

$$
x \text { is a smooth point of } Y(w) \text { if and only if } \# S_{x}(w)=\operatorname{dim} Y(w) \text {. }
$$

These reflections are in one-one correspondence with the $\mathfrak{T}$-stable curves in $Y(w)$ containing $x$ (see [5]). In fact, if $r=r_{\alpha}$ is the reflection associated to the root $\alpha \in \Phi(V)$, the root system of $(\mathrm{GL}(V), \mathfrak{T})$, and if $r x \neq x$, then either $\overline{U_{\alpha} x}$ or $\overline{U_{-\alpha} x}$ is a $\mathfrak{T}$-stable curve containing $x$ and $r x$. Here $U_{\alpha}$ denotes the one-dimensional unipotent group normalized by $\mathfrak{T}$ whose Lie algebra has $\mathfrak{T}$-weight $\alpha$. Using these results, it is not hard to determine the singular locus of $Y(w)$.

Definition 3.1. The conjugate Meyer diagram $\Delta(w)$ of $w($ or $Y(w))$ is the Young diagram with $d$ rows whose $i$-th row consists of $d(n-1)+i-w_{i}$ boxes. 
Notice that $d(n-1)+i$ is the $i$-th entry of the unique $\mathfrak{B}$-fixed point $e \in G(d, V)$. Clearly $e \in I^{u}$ is also the unique $\mathcal{B}$-fixed point in $X\left(w^{s}\right)$. The maximal singularities (maximal with respect to the Bruhat-Chevalley order) then are given by the hooks of $\Delta(w)$ as follows. A hook $\mathcal{H}$ is a sequence of consecutive rows $R_{i}, R_{i+1}, \ldots, R_{i+k}$ of $\Delta(w)(k>0)$ such that for the length $\left|R_{j}\right|$ of row $R_{j}$ we have

$$
\left|R_{i}\right|>\left|R_{i+1}\right|=\left|R_{i+2}\right|=\cdots=\left|R_{i+k}\right|>\left|R_{i+k+1}\right|,
$$

where by convention $R_{d+1}$ is an empty row in case $i+k=d$. The element $w_{\mathcal{H}} \leq w$ is the unique element of $I$, such that $\Delta\left(w_{\mathcal{H}}\right)$ is obtained from $\Delta(w)$ by replacing the rows $R_{i}, R_{i+1}, \ldots, R_{i+k}$ by rows of equal length $\left|R_{i+1}\right|-1$. Equivalently, $w_{\mathcal{H}}$ is obtained from $w$ by replacing $w_{i}$ with $w_{i+k}+1$. It is well known that $w_{\mathcal{H}}$ is a singularity of $Y(w)$ (see for instance [17] for a more general result).

In the affine setting however, things are more complicated. Firstly, (2) need not hold. Secondly, it is more complicated to even describe the smooth $\widehat{T}$-fixed points in combinatorial terms.

Definition 3.2. Let $x \in I^{u}$. For $1 \leq i \leq n$, we define

$$
S_{i}(x)=\{j \in x \mid j \equiv i \bmod n\}
$$

the $n$-string through $i$ in $x$.

Denote $\ell_{i}(x):=\left|S_{i}(x)\right|$. Notice that if $x=\tau^{c}$ and $c=\left(c_{1}, c_{2}, \ldots, c_{n}\right)$, then $\ell_{i}(x)=d-c_{i}$. Define $h_{i}(x)$ as the minimal element of $S_{i}(x)$, if $S_{i}(x)$ is nonempty (thus, $h_{i}(x)$ is the "head" of the string), and $h_{i}(x)=d n+i$, if $S_{i}(x)$ is empty. (Note that $h_{i}(x)=i+c_{i} n$ ). It will be convenient to denote the unique integer between 1 and $n$ congruent to a given integer $i \bmod n$ by $[i]$; thus, any $x_{k} \in x$ satisfies $x_{k} \in S_{\left[x_{k}\right]}(x)$.

Let $\hat{\alpha} \in \widehat{\Phi}$. Then clearly $s_{\hat{\alpha}}(|x|)$ is again a sequence of integers.

Definition 3.3. We say $s_{\hat{\alpha}}$ is defined at $x$, if $s_{\hat{\alpha}}(|x|)=|y|$ for some $y \in I^{u}$.

Note that this is equivalent to saying that $s_{\hat{\alpha}} x \leq w^{s}$. If $s_{\hat{\alpha}}$ is defined at $x$, then it operates on the strings, that is $s_{\alpha} x$ is obtained from $x$ by removing a number of elements in $S_{i}(x)$ and adding the same number to another string $S_{j}(x)$. Thus, $s_{\hat{\alpha}} x$ is determined by the requirements that $\ell_{i}\left(s_{\alpha} x\right)=\ell_{i}(x)-k$ and $\ell_{j}\left(s_{\hat{\alpha}} x\right)=\ell_{j}(x)+k$ for some suitable $k \geq 0$. The indices $i$ and $j$ are referred to as the indices corresponding to $s$. The simplest way to describe these operations is by means of the following diagram:

Definition 3.4. Let $x \in I^{u}$. The string diagram $\Sigma(x)$ of $x$ consists of $n$ rows where the $i$-th row has $\ell_{i}(x)$ boxes.

Obviously $\Sigma(x)$ is just an encoding of $c \in \mathbb{Z}^{n}$ in the description of $x$ as $x=\tau^{c}$, and $\ell_{i}(x)=$ $d-c_{i}$. The total number of boxes in $\Sigma(x)$ is always $d$.

\subsection{Small reflections}

We shall denote a typical reflection in $\widehat{W}$ by $s$, though $s$ has also been used as a superscript in $w^{s}$. But, we believe, this will not create any mix-up; whenever necessary, we will be explicit about the particular reference. There is a special class of reflections, which will play a crucial role in our description of singularities.

Definition 3.5. Let $x \in I^{u}$, and let $s \in \widehat{W}$ be a reflection defined at $x$, such that $s x \neq x$. Then $s$ is called small, if and only if $\left|s\left(x_{k}\right)-x_{k}\right|<n$ for all $1 \leq k \leq d$. Otherwise, $s$ is called large. 

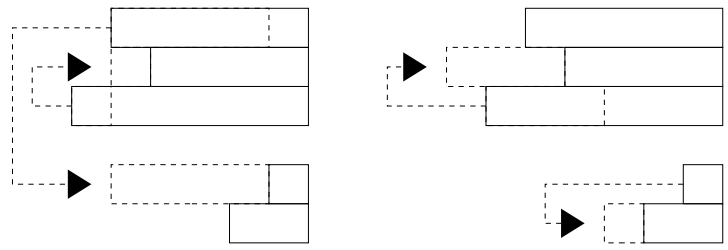

Figure 1. An example of $\Sigma(x)$ where $n=6, s=3, d=18, x=(\mathbf{7 5}, \mathbf{7 9}, 81,85, \mathbf{8 6}, 87,91,92$, $93,97,98,99, \mathbf{1 0 2}, 103,104,105,107, \mathbf{1 0 8}$ ) (the heads of the strings are in bold face), together with two small down-exchanges (left) and two large up-exchanges (right).

Remark 3.6. Let $s=s_{\hat{\alpha}}$ be any reflection defined at $x$ with $s_{\hat{\alpha}} x \neq x$. If $s_{\hat{\alpha}} x>x$ (respectively $s_{\hat{\alpha}} x<x$ ), there is a unique small reflection $s^{\prime}=s_{\hat{\alpha}^{\prime}}$ with $x<s^{\prime} x \leq s x$ (respectively, $x>s^{\prime} x \geq$ $s x$ ), and $\Re(\hat{\alpha})=\Re\left(\hat{\alpha}^{\prime}\right)$ (cf. Section 2.2). As an example, we treat the case $s_{\hat{\alpha}} x>x$. Suppose $\Re(\hat{\alpha})=(i j)$ with $i<j$, and given $\ell_{i}(x) \geq \ell_{j}(x)$, then $\hat{\alpha}^{\prime}=(i j)+\delta$. If $\ell_{i}(x)<\ell_{j}(x)$, then $\hat{\alpha}^{\prime}=(i j)$.

The small reflections are easily described in terms of $\Sigma(x)$ (see Fig. 1). For any pair of integers $1 \leq i<j \leq n$ there is almost always a unique small reflection $s$ with $s x>x$ and corresponding indices $i$ and $j$ : $s x$ is obtained from $x$ by $\ell_{i}(s x)=\ell_{j}(x)$ and $\ell_{j}(s x)=\ell_{i}(x)$ if $\ell_{j}(x)>\ell_{i}(x)$ (i.e. the rows of $\Sigma(x)$ at positions $i$ and $j$ are simply switched); if on the other hand $\ell_{i}(x) \geq \ell_{j}(x)>0$, then $s x$ satisfies $\ell_{i}(s x)=\ell_{j}(s x)-1$, and $\ell_{j}(s x)=\ell_{i}(x)+1$. The only case when $s$ does not exist is $\ell_{j}(x)=0$. We refer to the process of applying $s$ as up-exchanging $i$ and $j$.

Similarly, down-exchanging $i$ and $j$ is the inverse procedure, i.e. the result of down-exchanging $i$ and $j$ is the unique $x^{\prime}<x$, such that up-exchanging $i$ and $j$ in $x^{\prime}$ gives $x$. If $\ell_{i}(x)=\ell_{j}(x)$ or if $\ell_{i}(x)=\ell_{j}(x)-1$, then $x^{\prime}$ is not defined in this manner, and we let $x^{\prime}=x$, in this case.

To simplify our notation and get rid of the two different cases when up-exchanging (or downexchanging) we make a definition:

Definition 3.7. Define

$$
L(x)=\left(\ell_{1}(x), \ell_{2}(x), \ldots, \ell_{n}(x), \ell_{1}(x)+1, \ell_{2}(x)+1, \ldots, \ell_{n}(x)+1\right) .
$$

Notice that $x$ is uniquely determined by any $n$ consecutive entries of $L(x)$ (together with the first entry).

Let $L(x)=\left(l_{1}, \ldots, l_{2 n}\right)$. Up/down-exchanging $i$ and $j$ for $1 \leq i, j \leq 2 n$ always refers to up/down-exchanging $[i]$ and $[j]$. Suppose we want to up-exchange $i<j \leq n$. By replacing $i$ with $i+n$ and switching $i$ and $j$ if necessary, we may assume that $i<j$ and $l_{i}<l_{j}$ with $i \leq n$. If we now define $x$ by the $n$ entries $l_{i}, \ldots, l_{i+n-1}$, then the corresponding entries in $L(s x)$ are the same with the exception that $l_{i}$ and $l_{j}$ switch positions. We will often describe $x$ by any $n$ entries in $L(x)$ which contain $l_{i}$ or $l_{i+n}$ for every $1 \leq i \leq n$. Similarly, down-exchanging $[i]$ and $[j]$ reduces to switching the positions of $l_{i}$ and $l_{j}$ if we pick $i$ and $j$ such that $i<j$ and $l_{i}>l_{j}$. We write $i \downarrow j$ (resp. $i \uparrow j$ ) for the down-exchange (resp. up-exchange) of $i$ and $j$.

\subsection{The codimension of $X(s x)$ in $X(x), s x<x$}

Recall that a property of the Bruhat-Chevalley ordering is the fact that for any $x \leq w$ we have $\operatorname{codim}_{X(w)}(X(x))=\max \left\{i \mid \exists x=\tau_{0}<\tau_{1}<\cdots<\tau_{i}=w\right\}$. This still holds in the affine Grassmannian (easily proven combinatorially, or by the fact that the $\mathcal{B}$-orbits are isomorphic to affine spaces).

One reason for introducing $L(x)$ is the fact that if $s$ is a small reflection and $s x<x$, the codimension of $X(s x)$ in $X(x)$ may be read off immediately, thanks to the following lemma: 


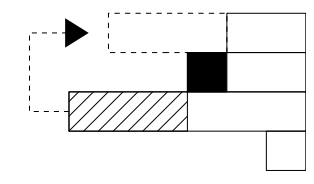

Figure 2. Here $n=4$, and 1 and 3 (resp. 3 and 5) are down-exchanged. The black box marks the entry in $x$ and $s x$ from which on they coincide. Since $g_{\geq}=0$, this is a codimension one down-exchange.

Lemma 3.8. Let $x \in I^{u}$ and let $s$ be a small reflection with $s x<x$. If sx is the result of down-exchanging $i<j<i+n$ with $l_{i}>l_{j}$ and $i<n$, say, then the codimension of $X(s x)$ in $X(x)$ equals $1+g_{\geq}+g_{>}$where

$$
g_{\geq}=g_{\geq}(i, j, x)=\left|\left\{i<k<j \mid L(x)_{i} \geq L(x)_{k} \geq L(x)_{j}\right\}\right|
$$

and

$$
g_{>}=g_{>}(i, j, x)=\mid\left\{i<k<j \mid L(x)_{i}>L(x)_{k}>L(x)_{j}\right\} .
$$

Notice that the assumptions $i<j$ and $L(x)_{i}>L(x)_{j}$ are no restriction as we may replace $i$ or $j$ if necessary with $i+n$ or $j+n$, respectively.

Proof. We proceed by induction on $g_{\geq}$. First suppose $g_{\geq}=0$. Then we have to show that $s x$ has codimension one. Equivalently, if $s x \leq y \leq x$, then either $y=x$ or $y=s x$. Suppose such a $y$ is given. We may describe $x, y$, and $s x$ by the entries of $L(\cdot)_{i+k}$ for $k=0,1, \ldots, n-1$.

First observe that $x$ and $s x$ coincide before (and including) the entry preceding $h_{i}(x)$ in $x$ and after (and including) the position of $h_{i}(s x)$ in $s x$. This immediately shows that $L(x)_{i} \geq L(y)_{i} \geq$ $L(s x)_{i}$. Similarly, $x$ and $s x$ coincide after the position of $h_{j}(x)-n$ in $s x$. Thus $L(y)_{j} \geq L(x)_{j}$. Now, if $L(y)_{j}>L(s x)_{j}=L(x)_{i}$, then $h_{j}(y)<h_{i}(x)$ and so would be in a range where $x$ and $s x$ coincide, a contradiction. Thus $L(s x)_{j} \geq L(y)_{j} \geq L(x)_{j}$.

For $k \neq i, j$, it follows that $L(y)_{k}=L(x)_{k}=L(s x)_{k}$ : indeed, suppose first that $i<k<j$; then as $h_{[k]}(x)<h_{i}(x)$ or $h_{[k]}(x)>h_{j}(x)$ for such $k$ (in view of the hypothesis that $g_{\geq}=0$ ), the position of $h_{[k]}(x)$ is in a range where $x$ and $s x$ agree. Moreover, $h_{[x]}(x)-n$ cannot be present in $y$, as it is not present in either of $x$ and $s x$, and would also fall into a range where $x$ and $s x$ agree (being strictly bigger than $h_{j}(x)-n$ ). Consequently, $h_{[k]}(y)=h_{[k]}(x)$, and it must have the same position.

Similarly, if $k>j$, and suppose $y$ contains $h \equiv k \bmod n$. Pick $r<h$ maximal congruent $i$. Then there is $t \equiv j \bmod n$ such that $r<t<h<r+n$. There are two cases: either $r$ is present in $x$, and $t$ is not, in which case $r$ is replaced by $t$ in $s x$, and the entries in $x$ and $s x$ strictly between $t$ and $r+n$ coincide and don't change positions; or, both $r$ and $t$, or neither of them, is present in $x$. In which case $x$ and $s x$ coincide at values strictly between $r$ and $r+n$ (including positions). It thus follows that $h$ must be in $x$ and $s x$. Consequently $h_{[k]}(y)=h_{[k]}(x)$ for all these $k$ (at the same position) and therefore $L(y)_{k}=L_{k}(x)=L_{k}(s x)$.

Since the coordinate sums of $L(y), L(x)$, and $L(s x)$ all agree, it follows that $L_{i}(y)+L_{j}(y)=$ $L_{i}(s x)+L_{j}(s x)=L_{i}(x)+L_{j}(x)$. If $L_{i}(x)>L_{i}(y)>L_{i}(s x)$, then necessarily $L_{j}(y)<L_{j}(s x)$, and the number of elements in $y$ that are less than or equal to $h_{j}(s x)$ is strictly smaller than the same number for $s x$, contradicting $y \geq s x$. The only two possibilities now are $L_{i}(y)=L_{i}(s x)$ or $L_{i}(y)=L_{i}(x)$, resulting in $y=s x$ or $y=x$ as claimed. This completes the proof in the case $g_{\geq}=0$.

Let then $g_{\geq}>0$, and let $i^{\prime}$ be the first index greater than $i$ such that $l_{i} \geq l_{i^{\prime}} \geq l_{j}$, then clearly $x \geq y \geq s x$, where $y$ is the result of $i \downarrow i^{\prime}$ (if $l_{i}=l_{i^{\prime}}$, then $x=y$ ): sx is obtained from $y$ by $i^{\prime} \downarrow j$, then $i \downarrow i^{\prime}$. Notice that the last down-exchange is necessary only if $L(x)_{i^{\prime}}>L(x)_{j}$. Consider the down-exchange of $i^{\prime}$ and $j$ in $y$, resulting in $y^{\prime} \geq s x$. Clearly $g_{\geq}\left(i^{\prime}, j, y\right)=g_{\geq}(i, j, x)-1$ 

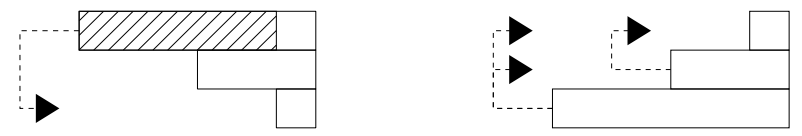

Figure 3. A down-exchange of 1 and 3 (left) and the corresponding reflections (right). Notice that here $g_{\geq}$and $g_{>}$both are 1 .

$\left(L(y)_{i^{\prime}}=L(x)_{i}\right)$. By induction, the codimension of $y^{\prime}$ in $X(y)$ is $1+g_{\geq}\left(i^{\prime}, j, y\right)+g_{>}\left(i^{\prime}, j, y\right)$. In addition, $g_{>}\left(i^{\prime}, j, y\right)=g_{>}(i, j, x)$ if $y=x$, and $g_{>}\left(i^{\prime}, j, y\right)=g_{>}(i, j, x)-1$ otherwise. If $y^{\prime} \neq s x$, then the codimension of $s x$ in $X\left(y^{\prime}\right)$ is 1 by construction and the application of the case $g_{\geq}=0$. Similarly, if $y \neq x$ then the codimension of $y$ in $X(x)$ is one as well. The result now follows.

One caveat is the following subtlety: strictly speaking, applying the induction hypothesis requires $i^{\prime} \leq n$. However, if $i^{\prime}>n$ the numbers $g_{>}\left(i^{\prime}, j, y\right)$ and $g_{\geq}\left(i^{\prime}, j, y\right)$ do not change if $i^{\prime}$ and $j$ are replaced with $i^{\prime}-n$ and $j-n$.

Remark 3.9. Suppose $x, s, i$ and $j$ are as in Lemma 3.8. Notice that an element of $G_{\geq}(i, j, x):=$ $\left\{i<k<j \mid L(x)_{i} \geq L(x)_{k} \geq L(x)_{j}\right\}$ gives rise to either one or two reflections $s^{\prime}$ with $x>s^{\prime} s x>s x$ as follows: If $L(x)_{i}>L(x)_{k}$ up-exchanging $j$ and $k$ in $s x$ is possible and the result is below $x$, because it is obtained from $x$ by $k \downarrow j$ and then $i \downarrow k$. If on the other hand $L(x)_{k}>L(x)_{j}$, then up-exchanging $k$ and $i$ is possible in $s x$ and below $x$, because it is the same as $k \downarrow j$ and $i \downarrow j$ applied to $x$.

Thus there are precisely $g_{>}$elements of $G_{\geq}(i, j, x)$ giving rise to two reflections. The total number of reflections thus obtained including $s$ is $1+g_{\geq}+g_{>}$, the codimension of $s x$ in $x$. We refer to these reflections as the reflections corresponding to the down-exchange of $i$ and $j$. For an example see Fig. 3.

\section{The connection between $X(w)$ and $Y(w)$}

In this section we will further investigate the connection between $X(w) \subset Y(w)$. One might think that $X(w)=Y(w)^{u}$ also as a scheme, but as it turns out this is not true in general, as it may happen that $T_{x}(Y(w))^{u} \supsetneq T_{x}(X(w))$ at some $x \leq w$. Nevertheless, a first step in computing $T_{x}(X(w))$ is to determine $T_{x}(Y(w))^{u}$, and in some cases knowledge of the latter is enough to determine the former.

\subsection{Tangents to the Grassmannian}

We will need the following well known description of the tangent space to $T_{x}(G(d, V))$ at a point $x$. Viewing $x$ as a subspace of $V$, let $p_{x}: V \rightarrow V / x$ and $i_{x}: x \rightarrow V$ be the projection and inclusion maps, respectively.

Lemma 4.1. Let $x \in G(d, V)$. Then

$$
T_{x}(G(d, V))=\operatorname{Hom}(x, V / x)
$$

in a natural way. In addition, the differential of the orbit map $\mathrm{GL}(V) \rightarrow G(d, V)$ which sends $g$ to gx, is given by

$$
\begin{array}{ccc}
\operatorname{End}(V) & \rightarrow & \operatorname{Hom}(x, V / x), \\
\xi & \mapsto & p_{x} \xi i_{x} .
\end{array}
$$

If $P$ denotes the stabilizer of $x$ in $\mathrm{GL}(V)$, this map is equivariant with respect to the adjoint action of $P$ on $\operatorname{End}(V)$, and the natural action of $P$ on $\operatorname{Hom}(x, V / x)$. 
Remark 4.2. If $E_{i j}(1 \leq i, j \leq d n)$ denotes the element in End $V$, sending $e_{j}$ to $e_{i}$ and if $x=\left(x_{1}, x_{2}, \ldots, x_{d}\right) \in I$, then $T_{x}(G(d, V))$ is spanned by the images of those $E_{i j}$ for which $j \in x$ but $i \notin x$. We will denote these elements of $T_{x}\left(G(d, V)\right.$ by $E_{i j}$ as well. Notice that $E_{i j}$ is a $\mathfrak{T}$-eigenvector of weight $\epsilon_{i}-\epsilon_{j}$ (where $\epsilon_{k}$ is the element in the character group of $\mathfrak{T}$, sending a diagonal matrix in $\mathfrak{T}$ to its $k$-th diagonal entry). Thus, its $\widehat{T}$-weight is $\epsilon_{[i]}-\epsilon_{[j]}+h \delta$ for a suitable $h$, where either $[i] \neq[j]$ or $h \neq 0$. In particular, all the $\widehat{T}$-weights of $T_{x}(G(d, V))$ are roots.

\subsection{Real tangents}

It is now clear, that if $x$ is an element of $I^{u}$, then $\xi \in T_{x}(G(d, V))$ is $u$-fixed if and only if $\tau \xi=\xi \tau$. From this it follows easily that any $\xi \in T_{x}(G(d, V))^{u}$ is uniquely determined by its values on $e_{h_{i}(x)}(1 \leq i \leq n)$ such that $h_{i}(x) \leq d n$; for, then $\xi\left(e_{h_{i}(x)+r n}\right)=\tau^{r} \xi\left(e_{h_{i}(x)}\right)$, $0 \leq r<\ell_{i}(x)$.

Definition 4.3. Let $x \in I^{u}$, and suppose $\hat{\alpha}=\alpha+h \delta \in \widehat{\Phi}$. We may write $\alpha=(i j)$ for some $1 \leq i, j \leq n$. If $0<\ell_{j}(x)$ and $h_{j}(x)=j+t n$ such that $t+h \geq 0, i+(t+h) n<h_{i}(x)$, and $i+\left(t+h+\ell_{j}(x)\right) n \geq h_{i}(x)$, then $\xi_{\hat{\alpha}}$ in $\operatorname{Hom}(x, V / x)$ is defined as:

$$
\xi_{\hat{\alpha}} e_{k}= \begin{cases}p_{x} e_{i+(r+t+h) n}, & r>0, k=h_{j}(x)+r n . \\ 0, & \text { otherwise. }\end{cases}
$$

To avoid having to state the hypotheses over and over again, we simply say, $\xi_{\hat{\alpha}}$ is defined at $x$ to indicate that all conditions above are met.

Lemma 4.4. Suppose $\xi_{\hat{\alpha}}$ is defined at $x \in I^{u}$. Then $\xi_{\alpha}$ is a u-fixed $\widehat{T}$-eigenvector of $T_{x}(G(d, V))$ of weight $\hat{\alpha}$. Conversely, if $\xi$ is any $\widehat{T}$-eigenvector in $T_{x}(G(d, V))^{u}$ whose weight is a real root $\hat{\alpha}$, then $\xi_{\hat{\alpha}}$ is defined at $x$ and $\xi \in K \xi_{\hat{\alpha}}$.

Proof. The first statement is immediate from the definition; we will therefore prove the second assertion: Suppose $\xi \in T_{x}(G(d, V))^{u}$ has $\widehat{T}$-weight $\hat{\alpha}=\alpha+h \delta$. Again write $\alpha=(i j)$. Let $x_{r} \in S_{k}(x)$ (cf. Definition 3.2), $x_{r}=k+m n$, say. Then the $\widehat{T}$-weight of $e_{x_{r}}$ is $\epsilon_{k}+m \delta$. As $\xi e_{x_{r}}$ has weight $\epsilon_{k}+\alpha+(m+h) \delta$, and by the description of possible $\widehat{T}$-weights in $\operatorname{Hom}(x, V / x)$ (cf. Remark 4.2) it follows that $k$ must equal $j$, if $\xi e_{x_{r}}$ is not to be zero. Therefore $\xi$ is uniquely determined by its values at $e_{h_{j}(x)}$, and it is sufficient to show that $\xi_{\hat{\alpha}}$ is defined at $x$ and that $\xi e_{x_{r}} \in K \xi_{\hat{\alpha}} e_{x_{r}}=K e_{i+(h+m) n}$ in case $x_{r}=k+m n=h_{j}(x)$. But $e_{i+(h+m) n}$ is the only possibility of an element of $V / x$ with weight $\epsilon_{i}+(h+m) \delta$ (up to scalars). As $\xi$ is nonzero, it follows $\xi e_{h_{j}(x)}=c e_{i+(h+m) n}$ for some $c \neq 0$, and in particular $0 \leq(h+m)$ and $i+(h+m) n<h_{i}(x)$. Since $\xi$ is also $\tau$-equivariant we must have $\tau^{\ell_{j}(x)} \xi e_{h_{j}(x)}=0$, and thus $i+\left(h+m+\ell_{j}(x)\right) n \geq h_{i}(x)$. But now $\xi_{\hat{\alpha}}$ is defined at $x$ and obviously $\xi$ is proportional to $\xi_{\hat{\alpha}}$.

We will refer to the elements of $T_{x}(G(d, V))^{u}$ which have a real root as $\widehat{T}$-weight as real tangents. Notice that if $\xi_{\hat{\alpha}}$ is defined at $x$, and $\hat{\alpha}>0$, then $\xi_{\hat{\alpha}}$ actually lifts to a $\tau$-invariant element of $\operatorname{End}(V)$ of $\widehat{T}$-weight $\hat{\alpha}$. In fact, if $\hat{\alpha}=(i j)+h \delta>0$ (and in particular, $h \geq 0$ ) then

$$
\xi_{\hat{\alpha}}=E_{i+h n, j}+E_{i+(h+1) n, j+n}+\cdots+E_{i+(d-1) n, j+(d-h-1) n} \in \operatorname{End}(V)^{u} .
$$

If $h<0$ this is not possible (as then $j+(d-h-1) n>d n$, so no element of $V$ can be mapped to $\left.e_{i+(d-1) n}\right)$. But we still have

$$
\xi_{\hat{\alpha}}=\sum_{\substack{0 \leq r<d \\ j+r n \geq h_{j}(x)}} E_{i+(r+h) n, j+r n}
$$

in $\operatorname{Hom}(x, V / x)$ with the convention that $E_{i, j}=0$ if $i$ or $j>d n$. 


\subsection{Reflections and $\widehat{T}$-curves}

The main goal of this section is to show that actually $\xi_{\hat{\alpha}} \in T_{x}\left(X\left(w^{s}\right)\right)$ whenever $\xi_{\hat{\alpha}}$ is a real tangent defined at $x$. As noted in the previous section, the $\mathfrak{T}$-stable curves play a crucial role when determining the singularities of a classical Schubert variety. They still give rise to a necessary though not sufficient criterion in the case of affine Schubert varieties. Let $x \leq w \in I^{u}$. A $\widehat{T}$-curve through $x$ in $X(w)$ is the closure of a one-dimensional $\widehat{T}$-orbit in $X(w)$ which contains $x$. We denote the set of all $\widehat{T}$-curves through $x$ by $E(X(w), x)$. By results of [5],

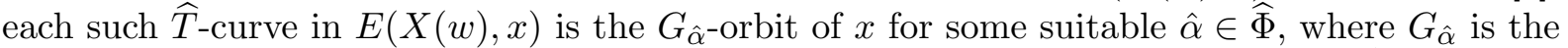
copy of $\mathrm{SL}_{2}(K)$ in $\mathcal{G}$ which is generated by the root-groups $U_{ \pm \hat{\alpha}}$; here, for any $\hat{\alpha} \in \widehat{\Phi}, U_{\hat{\alpha}}$ is the (uniquely determined) image of an inclusion $x_{\hat{\alpha}}: K \rightarrow \mathcal{G}$ that is equivariant with respect to the $\widehat{T}$-actions $(\widehat{T}$ acts on $K$ by $\hat{\alpha}$ and by conjugation on $\mathcal{G}): x_{\hat{\alpha}}(\hat{\alpha}(t) k)=t x_{\hat{\alpha}}(k) t^{-1}$ (conjugation here means that $t=\left(t_{0}, s\right) \in \widehat{T}=T \times K^{*}$ acts as $\left.\left.\operatorname{tgt}^{-1}:=s t_{0} g t_{0}^{-1}\right)\right)$. It follows that around $x$, any such $C$ has the form $U_{\hat{\alpha}} x$ for a suitable $\hat{\alpha}$, and $T_{x}(C)$ is a line in $T_{x}(X(w))$ with $\widehat{T}$-weight $\hat{\alpha}$. In particular $C$ is smooth. Moreover only real roots occur as weights. Let $T E(X(w), x))$ denote the "span" of the $\widehat{T}$-curves, that is,

$$
T E(X(w), x)=\bigoplus_{C \in E(X(w), x)} T_{x}(C)
$$

An immediate consequence of Lemma 2.2 is the fact that $|E(X(w), x)| \geq \operatorname{dim} X(w)$ (for a proof see [5]). This is sometimes referred to as Deodhar's inequality. As no two $\widehat{T}$-curves have the same $\widehat{T}$-weight at $x$, it follows that $\operatorname{dim} T E(X(w), x) \geq \operatorname{dim} X(w)$. Summarizing, let us recall the following necessary criterion from [5] for $x \leq w$ being a smooth point of $X(w)$ :

Lemma 4.5. Let $x \leq w$; if $x$ is a smooth point of $X(w)$, then

$$
|E(X(w), x)|=\operatorname{dim} X(w) .
$$

Equivalently, the number of reflections $s$ such that $s x \neq x$ and $s x \leq w$ equals $\operatorname{dim} X(w)$. This in turn is equivalent to

$$
|\{s \mid x<s x \leq w\}|=\operatorname{codim}_{X(w)}(X(x)) .
$$

The last statement of the Lemma is an immediate consequence of the fact that $|E(X(x), x)|=$ $\operatorname{dim} X(x)$, as $x$ is a smooth point of $X(x)$.

It should be pointed out, however, that contrary to the classical setting, this condition is not sufficient (see Remark 4.19).

One of the reasons that smoothness of Schubert varieties in the affine Grassmannian is a more delicate question than in the ordinary Grassmannian, is the existence of imaginary roots. For instance we have:

Lemma 4.6. Let $x \leq w \in I^{u}$. If there is a line $L \subset T_{x}(X(w))$ which is a $\widehat{T}$-eigenvector whose weight is an imaginary root, then $x$ is a singular point.

Proof. By the remarks preceding the lemma, the $\widehat{T}$-weights of $T E(X(w), x)$ are real roots. Thus, $L \neq T_{x}(C)$ for all $C \in E(X(w), x)$. The lemma now follows from the following well-known fact: If a torus $S$ acts on an affine variety $X$ with smooth fixed point $x$, then for every $S$-stable subspace $M \subset T_{x}(X)$ there exists an $S$-stable subvariety $X^{\prime} \subset X$ such that $T_{x}\left(X^{\prime}\right)=M$. In the case of $X=X(w)$, applying this to an open affine neighborhood of $x$, and putting $M=L$, the result follows. 
Remark 4.7. While it is true that $\xi_{\hat{\alpha}}$ is actually tangent to the $\widehat{T}$-curve $\overline{U_{\hat{\alpha}} x} \subset \mathcal{G} / \mathcal{P}$ it is not always possible to realize this identification inside $G(d, V)$, since $U_{\hat{\alpha}}$ may not act on $V$ (in particular if $\hat{\alpha}<0)$ commuting with $\tau$. If $\hat{\alpha}$ is positive, then $\xi_{\hat{\alpha}} \in \operatorname{End}(V)$ is nilpotent and actually spans the image of $\operatorname{Lie}\left(U_{\hat{\alpha}}\right)$ in $\operatorname{End}(V)$. Consequently, $U_{\hat{\alpha}} \subset \mathcal{B}$ injects into $\operatorname{GL}(V)$. In fact, let $U_{k l} \subset \mathrm{GL}(V)$ denote the root group with Lie algebra $K E_{k l}$. Then the image of $U_{\hat{\alpha}}$ is a one-dimensional subgroup of

$$
U:=U_{i+h n, j} U_{i+(h+1) n, j+n} \cdots U_{i+(d-1) n, j+(d-h-1) n} .
$$

Notice that all the individual factors in this product mutually commute, and that this product therefore is direct and a subgroup of $\operatorname{GL}(V)$, and $U_{\hat{\alpha}}=U \cap C(u)$ where $C(u)$ denotes the centralizer of $u$ in $\operatorname{GL}(V)$ (here, $u=1+\tau$ (cf. Section 2.4)).

Lemma 4.8. Let $\hat{\alpha} \in \widehat{\Phi}$. If $\hat{\alpha}>0$, then $\xi_{\hat{\alpha}}$ is defined at $x$ if and only if $s_{\hat{\alpha}} x<x$. If $\hat{\alpha}<0$, then $\xi_{\hat{\alpha}}$ is defined at $x$ if and only if $x<s_{\hat{\alpha}} x \leq w^{s}$.

As a consequence, $\xi_{\hat{\alpha}} \in T E\left(X\left(w^{s}\right), x\right)$, if it is defined.

Proof. If $\hat{\alpha}>0$, and $\xi_{\hat{\alpha}}$ is defined at $x$ then by the remarks preceding the lemma, $U_{\hat{\alpha}} \subset \mathrm{GL}(V)$, and $U_{\hat{\alpha}} x \neq x$. Since $\overline{U_{\hat{\alpha}} x}$ is a $\widehat{T}$-curve connecting $x$ and $s_{\hat{\alpha}} x$, the result follows.

If $\hat{\alpha}<0$, then the conditions for $\xi_{\hat{\alpha}}$ to be defined at $x$ assert that $x<s_{\hat{\alpha}} x \leq w^{s}$. Then $C:=\overline{U_{-\hat{\alpha}} s_{\hat{\alpha}} x}$ is a $\widehat{T}$-curve of $X\left(w^{s}\right)$ containing $x$. Since ker $\hat{\alpha} \subset \widehat{T}$ acts trivially on this curve, its tangent lines must have $\widehat{T}$-weights in $\mathbb{Q} \hat{\alpha} \subset X(\widehat{T}) \otimes \mathbb{Q}$. Obviously, $\hat{\alpha}$ is the only $\widehat{T}$-weight of $T_{x}(G(d, V))^{u}$ satisfying this condition, and the only corresponding eigenvector is $\xi_{\hat{\alpha}}$. As a consequence $T_{x}(C)=K \xi_{\hat{\alpha}} \subset T E\left(X\left(w^{s}\right), x\right)$.

If for any $\widehat{T}$-stable subspace $M \subset T_{x}(G(d, V)), M_{\text {re }}$ denotes the span of weight-subspaces for real roots, then we have seen:

Corollary 4.9. For any $x \leq w^{s}$ we have

$$
T_{x}\left(X\left(w^{s}\right)\right)_{\mathrm{re}}=T E\left(X\left(w^{s}\right), x\right)=T_{x}(G(d, V))_{\mathrm{re}}^{u} .
$$

For general $w$ the situation is more delicate. One might think that $T_{x}(X(w))_{\mathrm{re}}=T_{x}(Y(w))_{\mathrm{re}}^{u}$, but this is not true in general. Also it is not clear whether $T E(X(w), x)=T_{x}(X(w))_{\text {re }}$.

Remark 4.10. Let $x, w \in I^{u}, x \leq w$. Suppose that $\xi_{\hat{\alpha}}$ is defined at $x$ for $\hat{\alpha}=(i j)+h \delta$. Recalling (cf. equation (4)) that $\xi_{\hat{\alpha}} \in T_{x}(Y(w))^{u}$ if and only if $E_{i+(k+h) n, j+k n} \in T_{x}(Y(w))$ for all $k$ such that $0<i+(k+h) n<h_{i}(x)$ and $k<d$. Indeed, as $T_{x}(Y(w))$ is T-stable, $\xi_{\hat{\alpha}}$ is contained in $T_{x}(Y(w))$ if and only if every $\mathfrak{T}$-eigenvector it is supported in is an element of $T_{x}(Y(w))$. This in turn is equivalent to saying that $r_{i+(k+h) n, j+k n} x \leq w$ for all such $k$, where $r_{p q}$ denotes the transposition $(p q)$ in $\mathfrak{S}_{d n}=\mathfrak{W}$. Notice that all these $r_{i+(k+h) n, j+k n}$ commute.

Let $k_{0}$ be the maximal $k$ appearing in equation (4). Then $s_{\hat{\alpha}} x \leq w$ if and only if

$$
r_{i+h n, j} r_{i+(1+h) n, j+k n} \cdots r_{i+\left(k_{0}+h\right) n, j+k_{0} n} x \leq w
$$

a stronger condition than having just $r_{i+(k+h) n, j+k n} x \leq w$ for all $0 \leq k \leq k_{0}$ (at least for negative $\hat{\alpha}$ ). If $s_{\hat{\alpha}}$ is small however, the situation is different. Keeping, the notation just introduced, we have:

Lemma 4.11. Let $s_{\hat{\alpha}}$ be a small reflection (such that $\xi_{\hat{\alpha}}$ or $\xi_{-\hat{\alpha}}$ is defined at $\left.x \leq w\right)$. The following are equivalent:

1) $s_{\hat{\alpha}} x \leq w$; 
2) $r_{i+h+k n, j+k n} x \leq w$ for all $0 \leq k \leq k_{0}$;

3) $\xi_{\hat{\alpha}}$ or $\xi_{-\hat{\alpha}}$ is tangent to $X(w)$;

4) $\xi_{\hat{\alpha}}$ or $\xi_{-\hat{\alpha}}$ is tangent to $Y(w)$.

Proof. There is nothing to show if $s_{\hat{\alpha}} x<x$. So assume $s_{\hat{\alpha}} x>x$ and $\hat{\alpha}<0$. If $s_{\hat{\alpha}}$ is small, then the positions where $r_{i+(h+k) n, j+k n} x$ and $r_{i+\left(h+k^{\prime}\right), j+k^{\prime} n} x$ differ from $x$, are disjoint intervals in $[1, d]$. Consequently, $s_{\hat{\alpha}} x \leq w$ if and only if $r_{i+(h+k), j+k n} x \leq w$ for all $k=0,1, \ldots, k_{0}$. The latter condition is in turn equivalent to the fact that $\xi_{\hat{\alpha}} \in T_{x}(Y(w))$. Since $\xi_{\hat{\alpha}} \in T_{x}(X(w)$ always implies $\xi_{\hat{\alpha}} \in T_{x}(Y(w))$ the lemma now follows.

\subsection{Imaginary tangents}

Consistent with the notation introduced above, we call a tangent $\xi \in T_{x}(G(d, V))^{u}$ imaginary, if it is a $\widehat{T}$-eigenvector for an imaginary root. The Weyl group $\widehat{W}$ fixes the imaginary roots $\mathbb{Z} \delta$ identically, i.e. $w(\delta)=\delta$ for all $w \in \widehat{W}$. Since for $x=e \mathcal{P}$, the set of $\widehat{T}$-weights of $T_{x}(\mathcal{G} / \mathcal{P})$ does not contain any positive imaginary roots, this means the same applies at any $\widehat{T}$ fixed point $x \in \widehat{W} e \mathcal{P}$, as the weights at $x$ are the $\widehat{W}$-translates of the weights at $e \mathcal{P}$. But this may be seen directly as well: Let $\xi \in T_{x}(G(d, V))^{u}$ be an imaginary tangent of weight $h \delta$, say. Weight considerations then yield $\xi e_{k}=e_{k+h n}$, for $e_{k} \in x$, if $\xi e_{k} \neq 0$. Of course, $x$ contains $e_{k+h n}$ if $h \geq 0$, thus necessarily $h<0$ if $\xi \neq 0$.

Definition 4.12. Let $x \leq w^{s} \in I^{u}$. For any $i$ between 1 and $n$ and $h>0$ let $\xi_{i, h} \in T_{x}(G(d, V))$ be defined as follows, provided $h \leq \ell_{i}(x)$ and $h_{i}(x)-h n>0$ :

$$
\xi_{i, h} e_{k}= \begin{cases}p_{x} e_{k-h n}, & k \in S_{i}(x), \\ 0, & \text { otherwise. }\end{cases}
$$

Similar to the real case, we simply say $\xi_{i, h}$ is defined at $x$, if $h_{i}(x)-h n>0$.

Remark 4.13. It is clear that $\xi_{i, h}$ is $u$-invariant. It is also clear, that every imaginary tangent $\xi$ of weight $-h \delta$ is a linear combination of those $\xi_{i, h}$ which are defined at $x$ : As remarked above, $\xi$ is $u$-invariant and determined by its values on the various $e_{h_{i}(x)}$, and $\xi e_{h_{i}(x)}=\lambda_{i} e_{h_{i}(x)-h n}$, if $h_{i}(x)-h n>0$, and zero otherwise. Also, $\xi e_{h_{i}(x)}=0$ if $h>\ell_{i}(x)$, for then $\tau^{\ell_{i}(x)} \xi e_{h_{i}(x)} \neq 0$. Thus, $\xi=\sum_{i} \lambda_{i} \xi_{i, h}$ provided we defined $\lambda_{i} \xi_{i, h}$ to be zero if $h_{i}(x)-h n \leq 0$ or $\ell_{i}(x)<h$.

Our goal now is to describe those imaginary tangents which actually appear in $T_{x}\left(X\left(w^{s}\right)\right)$. To this end, let us keep $h>0$ fixed throughout the remainder of this subsection, and put

$$
S(h)=S(h, x)=\left\{i \mid h_{i}(x)-h n>0 ; h \leq \ell_{i}(x)\right\},
$$

the set of indices for which $\xi_{i, h}$ is defined. Clearly,

$$
T_{x}(G(d, V))_{-h \delta}^{u}=\bigoplus_{i \in S(h)} K \xi_{i, h}
$$

is the weight space of imaginary weight $-h \delta$. Before we describe the tangents belonging to $T_{x}\left(X\left(w^{s}\right)\right)$ we need some more notation. Recall the notion of Plücker coordinates on $G(d, V)$ : for $x \in I$, let $p_{x}$ be the corresponding Plücker coordinate (equal to $e_{x_{1}}^{*} \wedge e_{x_{2}}^{*} \wedge \cdots \wedge e_{x_{d}}^{*}$, with the $e_{i}^{*}$ being a dual basis for the $\left.e_{i}\right)$. The $p_{x}$ generate the homogenous coordinate ring of $G(d, V)$ (for the Plücker embedding $G(d, V) \hookrightarrow \mathbb{P}\left(\bigwedge^{d} V\right)$ ). For $x \in I$, let $U_{x}$ denote the open set $p_{x} \neq 0$ in $G(d, V)$. Then $U_{x}$ is open affine, $\mathfrak{T}$-stable (and actually isomorphic to a $\mathfrak{T}$-module). For any $\theta \in I, f_{\theta}=\frac{p_{\theta}}{p_{x}}$ is a well defined function on $U_{x} ; \mathcal{O}\left(U_{x}\right)$ is generated by those $f_{\theta}$ which have 
nonzero differential at $x$. The $\theta$ 's in $I$ for which this holds are precisely those, which differ in exactly one entry from $x$, that is, $\theta=r_{i, j} x$ for suitable $i, j$. Here $r_{i, j} \in \mathfrak{W}=\mathfrak{S}_{d n}$ denotes the reflection exchanging $i$ and $j$. For any $E_{k l} \in T_{x}(G(d, V))$ we have $d f_{\theta, x}\left(E_{k l}\right)=(-1)^{d(\theta)} \delta_{i k} \delta_{j l}$, where $d(\theta)$ is the difference in positions between $j$ in $x$ and $i$ in $\theta$. Let $\theta_{i, r}$ be obtained from $x$ by replacing $h_{i}(x)+r n$ with $h_{i}(x)+(r-h) n$, provided $0 \leq r<h \leq \ell_{i}(x)$ and $h_{i}(x)+(j-h) n>0$.

Lemma 4.14. On $T_{x}\left(X\left(w^{s}\right)\right)$ we have

$$
\sum_{i \in S(h, x)}(-1)^{d\left(\theta_{i, 0}\right)} d f_{\theta_{i, 0}, x}=0
$$

Proof. Consider the action of $\tau^{h}$ on $\bigwedge^{d} V$ and $\bigwedge^{d} V^{*}$ (acting as

$$
v_{1} \wedge v_{2} \wedge \cdots \wedge v_{d} \mapsto \sum_{i=1}^{d} v_{1} \wedge \cdots \wedge \tau^{h}\left(v_{i}\right) \wedge \cdots \wedge v_{d}
$$

and similarly for $\bigwedge^{d} V^{*}$ ). If $M \subset V$ is a $d$-dimensional subspace normalized by $u, \bigwedge^{d} M$ is in the kernel of $\tau^{h}$ (as $\tau^{h}$ acts nilpotently on $M$ ). Thus, for every $w \in I, p_{w} \in \bigwedge^{d} V^{*}$ satisfies $p_{w} \tau^{h}(M)=0$, and consequently $\tau^{h} p_{w}$ vanishes on $G(d, V)^{u}$. In particular $\tau^{h} p_{x}=0$ on $G(d, V)^{u}$. A straight forward computation (keeping in mind that $\tau^{h} e_{k}^{*}=-e_{k-h n}^{*}$ ) shows that

$$
\tau^{h} p_{x}=\sum_{i=1}^{n} \sum_{\substack{0 \leq j<\ell_{i}(x) \\ 0<h_{i}(x)+(j-h) n<h_{i}(x)}}(-1)^{d\left(\theta_{i, j}\right)} p_{\theta_{i, j}}+R,
$$

where $R$ is a linear combination of Plücker coordinates $p_{w}$ where $w$ differs from $x$ in strictly more than one element. Localizing to $x$, we therefore obtain a relation

$$
\sum_{i} \sum_{\substack{0 \leq j<\ell_{i}(x) \\ 0<h_{i}(x)+(j-h) n<h_{i}(x)}} d f_{\theta_{i, j}, x}=0
$$

on $T_{x}\left(X\left(w^{s}\right)\right)$. Every summand of this relation has $\widehat{T}$-weight $-h \delta$. To prove the lemma it therefore suffices to consider the relation evaluated on $T_{x}(G(d, V))_{-h \delta}^{u}$. Here, though, $d f_{\theta_{i, j}, x}=$ $(-1)^{d\left(\theta_{i, j}\right)-d\left(\theta_{i, 0}\right)} d f_{\theta_{i, 0}, x}$ by the definition of $\xi_{i, h}$, if $0<h_{i}(x)-h n$. If on the other hand $h_{i}(x)-$ $h n=0$, then $T_{x}(G(d, V))_{-h \delta}^{u}$ contains no element supported in $E_{k, i+r n}$ for all $k$ and $r$, and therefore $d f_{\theta_{i, j}, x}=0$ in $T_{x}(G(d, V))_{-h \delta}^{u}$. Summarizing, we get $\sum_{i=1}^{n} h(-1)^{d\left(\theta_{i, 0}\right)} d f_{\theta_{i, 0}, x}=0$, and the result follows.

Notice that $d f_{\theta_{i, 0}, x}\left(\xi_{i, h}\right)=(-1)^{d\left(\theta_{i, 0}\right)}$. Thus, $\sum_{i \in S(h)} c_{i} \xi_{i, h} \in T_{x}\left(X\left(w^{s}\right)\right)$ implies $\sum_{i} c_{i}=0$. We refer to the relations of Lemma 4.14 as trace relations. The reason for this is that when considering the open immersion of the nullcone $\mathcal{N}$ of nilpotent matrices into $X\left(w^{1}\right)$ alluded to in the Introduction (cf. Lusztig's isomorphism [19]), 0 is sent to $e$, and these relation in case $h=1$ actually correspond to the vanishing of the trace on nilpotent matrices.

We will show below (cf. Theorem 6.9), that the trace relations are the only linear relations on $T_{x}\left(X\left(w^{s}\right)\right)_{-h \delta} \subset T_{x}(G(d, V))_{-h \delta}^{u}$.

Let $x, w \in I^{u}, x \leq w$. Similar to the case of real roots (cf. Remark 4.10), we have the following Lemma describing $T_{x}(Y(w))_{-h \delta}^{u}$. First one notation: Set

$$
S(h, x, w)=\left\{i \in S(h, x) \mid r_{h_{i}(x)+(j-h) n, h_{i}(x)+j n} x \leq w, \forall j=0,1, \ldots, h-1\right\} .
$$

(Here, $S(h, x)$ is as in equation (5).) 
Lemma 4.15. Let $x, w \in I^{u}, x \leq w$. Then

(1) $T_{x}(Y(w))_{-h \delta}^{u}$ is spanned by all $\xi_{i, h}$, defined at $x$, which are contained in $T_{x}(Y(w))$.

(2) $T_{x}(Y(w))_{-h \delta}^{u}=\bigoplus_{i \in S(h, x, w)} K \xi_{i, h}$.

Proof. Indeed, any $\xi \in T_{x}(Y(w))_{-h \delta}^{u}$ is a linear combination of $\xi_{i, h} \mathrm{~s}$, defined at $x$. On the other hand, the $\xi_{i, h} \mathrm{~s}$ are supported in entirely different $\mathfrak{T}$-eigenspaces. Thus, $\xi \in T_{x}(Y(w))^{u}$ can be supported in $\xi_{i, h}$ only if all the $\mathfrak{T}$-eigenvectors in which $\xi_{i, h}$ has a nonzero component and thus $\xi_{i, h}$ - are elements of $T_{x}(Y)$. Assertion (1) now follows.

Assertion (2) follows from Assertion (1) and the definition of $S(h, x, w)$.

As a consequence, we have the following

Theorem 4.16. $T_{x}\left(Y\left(w^{s}\right)\right)^{u}=T_{x}(G(d, V))^{u}$ for all $x \in I^{u}$.

Proof. It is easily seen that for all $x, S\left(h, x, w^{s}\right)=S(h, x)$. The result follows from this fact, the above lemma, equation (6), and Corollary 4.9.

Returning to our study of $T_{x}\left(X\left(w^{s}\right)\right)$ for some arbitrary but fixed $x \leq w^{s}$, we make the following

Definition 4.17. Let $i, j \in S(h, x)$ be arbitrary but distinct. For $\alpha=(i j) \in \Phi$ we set

$$
\xi_{\alpha, h}=\xi_{i, h}-\xi_{j, h} \in T_{x}(G(d, V))^{u} .
$$

Notice that by the trace relation, the tangents of the form $\xi_{\alpha, h}$ span $T_{x}(X(w))_{-h \delta}$.

Lemma 4.18. Suppose $s_{\hat{\alpha}}$ is a large reflection defined at $x \leq w \in I^{u}$ with $x<s_{\hat{\alpha}} x \leq w$. Then $x$ is a singular point of $X(w)$.

Proof. We will show that there is an imaginary tangent in $T_{x}(X(w))$. In fact, it will be $\xi_{\Re(\hat{\alpha}), 1}$ (recall $\Re(\hat{\alpha})$ from Section 2.2). By Lemma 4.6, the conclusion follows.

We may write $\hat{\alpha}=(i j)+h \delta$, with $h \leq-1$. Let $\hat{\beta}=\hat{\alpha}+\delta$. $\hat{\beta}$ is negative as well: if $(i j)<0$ this is clear; if $(i j)>0$ then $h \leq-2$ since $s_{\hat{\alpha}}$ is large, and $\hat{\beta}<0$ follows. There are two cases to consider: either $s_{\hat{\beta}} x$ is defined at $x$, and $s_{\hat{\beta}} x>x$, or $s_{\hat{\beta}} x=x$. In both cases $U_{-\hat{\beta}}$ is a subgroup of the stabilizer of $x$ in $\mathcal{B}$ and consequently acts on $T_{x}(X(w))$. Notice that the Lie algebra of $U_{-\hat{\beta}}$ is spanned by $\xi_{-\hat{\beta}} \in \operatorname{End}(V)$ (cf. equation (3)). A straight forward computation then shows that $\left[\xi_{-\hat{\beta}}, \xi_{\hat{\alpha}}\right]=-\xi_{(i j), 1}$ (see Fig. 4). As this is the action of $\operatorname{Lie} U_{-\hat{\beta}}$ on $T_{x}(X(w)$ ), it follows that $\xi_{(i j), 1} \in T_{x}(X(w))$.

Of course the lemma also follows immediately if one considers the fact that $\left[\hat{\mathfrak{g}}_{-\hat{\beta}}, \hat{\mathfrak{g}}_{\hat{\alpha}}\right] \subset \hat{\mathfrak{g}}_{-\delta}$ is nonzero and therefore a tangent of $T_{x}\left(X(w)\right.$ ) (since $\hat{\mathfrak{g}} \rightarrow T_{x}\left(\mathrm{GL}_{n}(F) / \mathrm{GL}_{n}(A)\right.$ ) is surjective with kernel $x \hat{\mathfrak{g}}_{+} x^{-1}$ where $\left.\hat{\mathfrak{g}}_{+}=\bigoplus_{\delta(\hat{\alpha}) \geq 0} \hat{\mathfrak{g}}_{\hat{\alpha}}\right)$.

Remark 4.19. Recall (cf. [16]) that in the classical setting in type $A$, a point $x \leq w$ is smooth in $X(w)$ if and only if there are precisely $\operatorname{dim} X(w)$ reflections $r$ such that $x \neq r x \leq w$. In the affine setting, this is no longer true; this description fails for example if one of these reflections is large.

However, using D. Peterson's ideas of deforming tangent spaces (see [6] for a discussion of this approach), it seems to be possible to show that if for all $y$ with $x \leq y \leq w$ we have $T E(X(w), y)$ has $\operatorname{dim} X(w)$ elements, and furthermore no reflection $r$ with $y<r y \leq w$ is large, then $x$ is a smooth point. 

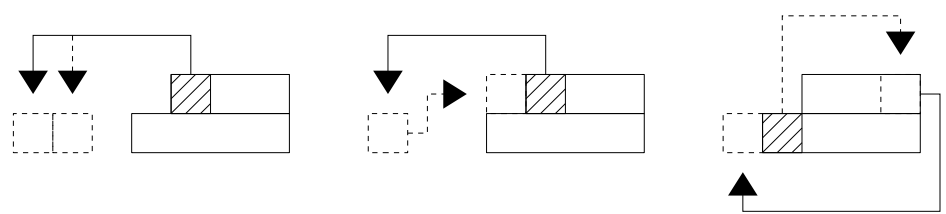

Figure 4. An example for how to create imaginary tangents as in the proof of Lemma 4.18. The left picture shows part of $\Sigma(w)$ and the effect of $s_{\hat{\alpha}}$ and $s_{\hat{\beta}}$ on the first box of the row (the arrows with the solid lines refer to $s_{\hat{\alpha}}$ ). The picture in the middle shows the effect of $\xi_{-\beta} \xi_{\hat{\alpha}}$ and the picture to the right shows $\xi_{\hat{\alpha}} \xi_{-\hat{\beta}}$.

\section{$5 \quad$ Real and imaginary patterns}

We are now ready to describe several types of singularities of a given $X(w) \subset X\left(w^{s}\right)$. As it turns out, the singularities are best described using $L(w)$, due to the subtlety that when down-exchanging $i>j$ (with $\ell_{i}(w)>\ell_{j}(w)$ ) the result is not just ex-changing the rows in $\Sigma(w)$.

\section{$5.1 \quad$ Imaginary patterns}

The previous section of course provides a very elementary way of producing singularities. For the sake of consistency we give it a name:

Definition 5.1. An imaginary pattern $P$ in $L(w)$ is a pair of integers $(i, j)(i<j)$ with $i \leq n$, such that $L(w)_{i}>L(w)_{j}+1$. For such a pattern $P$, let $w_{P}$ be obtained from $w$ by replacing $h_{i}(w)$ with $h_{j}(w)-n$.

Remark 5.2. Notice that $w_{P}$ is clearly singular, because it is of the form $s w<w$ with $s$ a large reflection.

In some cases all maximal singularities of a given Schubert variety arise in this fashion; for instance the single maximal singularity of $X\left(w^{s}\right)$ is $w^{s}{ }_{P}$ for $P=(12)$ (see Section 6).

Obviously, the condition of not admitting any imaginary pattern forms a serious obstruction against being non-singular. It is immediately forced that for a smooth $X(w), \ell_{i}(w) \leq \ell_{j}(w)+2$ for all pairs $i, j$, and $\ell_{i}(w)=\ell_{j}(w)+2$ is possible only if $j<i$.

\subsection{Real patterns}

Perhaps more interesting are the singularities which arise because $T E(X(w), x)$ is too large, or, in other words, because there are too many $\widehat{T}$-stable curves through $x$. Recall from Section 3 that the singularities of $Y(w)$ correspond to the hooks in $\Delta(w)$. This is no longer true for affine Schubert varieties, but there is a type of pattern in $L(w)$ which closely resembles this concept. In fact, a hook in $\Delta(w)$ is more or less a "gap" in $w$, i.e. an index $i$ such that $w_{i+1} \neq w_{i}+1$ together with a (first) position $k>i$ such that $w_{i+1}+(k-i) \neq w_{k+1}$. For $X(w)$ this is more complicated:

Definition 5.3. Let $w \in I^{u}$. A real pattern of the first kind in $L(w)$ is a sequence of integers $1 \leq i<g<j<k \leq 2 n$ subject to the following conditions:

1) $i<n, j<i+n$, and $k<g+n$;

2) $l_{i} \geq l_{j}>l_{g} \geq l_{k}$.

If $P=(i, g, j, k)$ is such a pattern, $w_{P}$ is obtained from $w$ by the following sequence of downexchanges: $i \downarrow g, g \downarrow j, g \downarrow k$. 

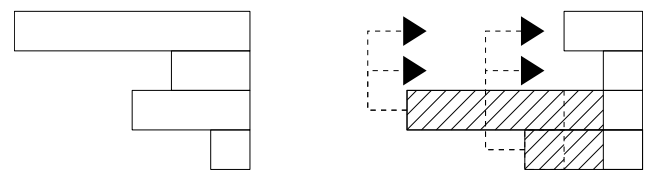

Figure 5. The basic example of a real pattern of the first kind. Here $P=(1,2,3,4)$, and $w_{P}$ is shown on the right, together with four up-exchanges. Notice that according to Lemma $3.8, w_{P}$ has codimension 3.
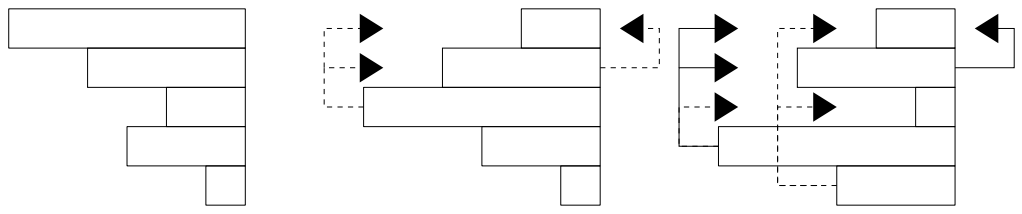

Figure 6. Another real pattern of the first kind: left $w$, in the middle $w_{1}$, and to the right $w_{P}$; here $P=(1,3,4,5)$. The up-exchanges constructed from the ones corresponding to $w_{1}<w$ have a solid line.

Proposition 5.4. If $P=(i, g, j, k)$ is a real pattern of the first kind in $L(w)$, then $w_{P}$ is singular. More precisely, if $k<i+n$, then $\left|E\left(X(w), w_{P}\right)\right|>\operatorname{dim} X(w)$. If $k>i+n$, then $w_{P}$ admits a large reflection $s$ such that $w_{P}<s w_{P} \leq w$.

It is worth mentioning, that $k=i+n$ does not occur, because $L(w)_{i}>L(w)_{k}$, which never holds for $k=i+n$. Before proving Proposition 5.4, notice first that

Remark 5.5. $w_{P}$ is determined by the requirements $L\left(w_{P}\right)_{i}=L(w)_{g}, L\left(w_{P}\right)_{g}=L(w)_{t}$, $L\left(w_{P}\right)_{j}=L(w)_{i}$, and finally $L\left(w_{P}\right)_{t}=L(w)_{j}$.

This is an immediate consequence of the fact, that for each down-exchange in the definition of $w_{P}$ the corresponding indices $i^{\prime}, j^{\prime}$ satisfy $i^{\prime}<j^{\prime}$ and $L\left(w^{\prime}\right)_{i^{\prime}}>L\left(w^{\prime}\right)_{j^{\prime}}$ where $w^{\prime}$ denotes the intermediate step on which the down-exchange is performed.

It should be mentioned that it is possible that Gasharov's proof of similar statements in the classical case [9] could be adapted to our situation to simplify the proofs of Proposition 5.4 as well as Proposition 5.7. However, except as mentioned below in Remark 5.8, we don't see how.

Proof of Proposition 5.4. Let $L(w)=\left(l_{1}, l_{2}, \ldots, l_{2 n}\right)$. First suppose that $k<i+n$. We will show that the number of reflections $s$ with $w_{P}<s w_{P} \leq w$ is too big, i.e. strictly larger than the codimension of $X\left(w_{P}\right)$ in $X(w)$ (see Lemma 4.5).

Let $w_{1}$ be obtained from $w$ by down-exchanging $i$ and $g$ (cf. Fig. 6). Then $w>w_{1}>w_{P}$. Let $c$ be the codimension of $w_{P}$ in $X\left(w_{1}\right)$. By Deodhar's Inequality there are at least $c$ reflections $s$ such that $w_{P}<s w_{P} \leq w_{1}$. Let $c_{1}$ be the codimension of $X\left(w_{1}\right)$ in $X(w)$. We will construct $c_{1}+1$ reflections $s$ with $w_{P}<s w_{P} \leq w$ but $s w_{P} \not \leq w_{1}$.

Consider the $c_{1}$ reflections corresponding to the down-exchange of $i$ and $g$ in $w$; such a reflection $s$ satisfies $w \geq s w_{1}>w_{1}$. According to Remark 3.9 there are three kinds of these reflections: first, an up-exchange of an element $h \in g_{\geq}(i, g, w)$ with $i$, and second an up-exchange of such an element with $[g]$. Finally, there is also the up-exchange of $i$ and $[g]$ (turning $w_{1}$ into $w$ ).

Now consider the first type. i.e. $s$ is an up-exchange of some $h \in g_{\geq}(i, g, w)$ with $i$, and then $w_{1}<s w_{1}<w$. Also, $i<h<g$ and $L(w)_{i} \geq L(w)_{h}>L(w)_{g}$. Notice that this means $[h] \neq[k]$; $[h] \neq[j]$ as well, because $i<h<j<i+n$. It follows that the very same up-exchange is defined at $w_{P}$ and $s w_{P}>w_{P}$. On the other hand $s w_{P} \leq w$, because it may be obtained by $g \downarrow j$, and then $g \downarrow k$ applied to $s w_{1}$. Also $s w_{P} \not w_{1}$ : of course, $w, w_{1}, w_{P}, s w_{P}$ all differ only in the $n$-strings through $i, h, g, j, k$ (in fact, this means, we may actually assume $n=5$ ); elements of these strings will be referred to as relevant. Moreover, $L\left(s w_{P}\right)_{i}=l_{h}$, so the elements of $s w_{P}$ in 

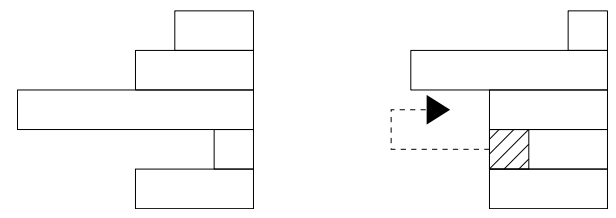

Figure 7. A real pattern of the first kind with $k>i+n . w$ is shown to the left, and $w_{P}$, together with the large up-exchange, is shown to the right. Assuming $n=5, P=(3,6,7,9)$.

these strings that are less than or equal to $h_{i}\left(s w_{P}\right)$ are given by $h_{i}\left(s w_{P}\right)$ itself, the first $l_{i}-l_{h}$ elements of $S_{[j]}\left(s w_{P}\right)$, and the first $l_{j}-l_{h}$ elements of $S_{[k]}\left(s w_{P}\right)$ (if $l_{j}>l_{h}$ ). Notice that if for any integer $r$ among $h, g, j, k$ we have $r>n$, then $[r]<i$ by assumption. So to see why $l_{i}-l_{h}$ elements of $S_{[j]}\left(s w_{P}\right)$ are less than or equal to $h_{i}\left(s w_{P}\right)$, observe that if $j \leq n$, then this is clear, as then also $h \leq n$. Otherwise, it follows that $[j]<i$ as $j<i+n$. Thus, exactly $l_{h}-1$ entries of $S_{[j]}\left(s w_{P}\right)$ are strictly larger than $h_{i}\left(s w_{P}\right)$. On the other hand, $L\left(s w_{P}\right)_{[j]}=l_{i}-1$, so the difference, i.e. the number of those less than or equal to $h_{i}\left(s w_{P}\right)$ is precisely $l_{i}-l_{h}$. The case of $[k]$ is similar. Notice that $k<i+n$ is needed here only if $l_{j}>l_{h}$.

In $w_{1}$, however, the relevant elements less than or equal to $h_{i}\left(s w_{P}\right)$ comprise only $l_{i}-l_{h}$ elements of $S_{[g]}\left(w_{1}\right)$ and possibly $l_{j}-l_{h}$ elements of $S_{[j]}\left(w_{1}\right)$ (if $l_{j}>l_{h}$ ). Thus the total number is strictly smaller, and $s w_{P} \not \leq w_{1}$.

The second possibility is that $s$ is an up-exchange of $h$ and $g$. In this case, let $s^{\prime}$ be the reflection associated to $j \uparrow h$ in $w_{P}$. As $L\left(w_{P}\right)_{h}<l_{i}$ in this case, $s^{\prime}$ is well-defined. Notice that this case also includes $h=i$. Moreover $s^{\prime} w_{P} \leq w$, as it may be obtained by $j \downarrow k$ and then $g \downarrow j$ in $s w_{1}$. Again $s w_{P} \not \leq w_{1}$, since the number of elements less than or equal to $h_{[h]}\left(s w_{P}\right)$ is strictly larger than the same number for $w_{1}$.

Summarizing, each of the $c_{1}$ reflections at $w_{1}$ gives rise to a reflection $s$ at $w_{P}$ such that $w_{P}<s w_{P} \leq w$, but $s w_{P} \not \leq w_{1}$.

It remains to construct one additional reflection with this property. Let $s_{1}$ be the reflection corresponding to $k \uparrow i$ in $w_{P}$. Clearly $s_{1} w_{P} \leq w$ as $s_{1} w_{P}$ may be obtained from $w$ by downexchanging $i$ and $j$, and $g$ and $k$ (which uses $k<i+n$ ). But again, $s_{1} w_{P} \not \leq w_{1}$ : the number of elements in $s_{1} w_{P}$ less than or equal to $h_{i}\left(s_{1} w_{P}\right)$ is strictly larger than the same number computed for $w_{1}$. In $w_{1}$ the only relevant such elements are the first $l_{i}-l_{j}$ elements of $S_{[g]}\left(w_{1}\right)$. The same number for $s_{1} w_{P}$, however, is given by 1 for $h_{i}\left(s_{1} w_{P}\right)$, plus the first $l_{i}-l_{j}$ elements of $S_{[j]}\left(s_{1} w_{P}\right)$.

Finally, in the case $k>i+n$, we will construct a large reflection: let $s$ correspond to two subsequent up-exchanges of $k$ and $i$ in $w_{P}$. Notice that $s$ is indeed large. $s w_{P}$ is obtained from $w_{P}$ by decreasing $L\left(w_{P}\right)_{k}$ by one, and increasing $L\left(w_{P}\right)_{i}$ by one $\left(k>n\right.$, hence $l_{k}>0$ and therefore $\left.L\left(w_{P}\right)_{i}=l_{g} \geq l_{k}>0\right)$. An example is outlined in Fig. 7 .

To recap, $s w_{P}$ is characterized by $L\left(s w_{P}\right)_{i}=l_{g}+1, L\left(s w_{P}\right)_{g}=L\left(w_{P}\right)_{g}=l_{k}, L\left(s w_{P}\right)_{j}=$ $L\left(w_{P}\right)_{j}=l_{i}$, and $L\left(s w_{P}\right)_{k}=l_{j}-1$. Thus, $s w_{P} \leq w$, as it is obtained from $w$ by $i \downarrow j$, and $g \downarrow k$, and, if $l_{j}>l_{g}+1, i \downarrow k$. As $s$ is large, $w_{P}$ must be a singular point of $X(w)$, as claimed.

The real patterns of the first kind are modeled loosely after the hooks in the classical setting, $h_{[g]}(w)-n$ playing the role of the "gap" between elements of $S_{i}(w)$ and $S_{[j]}(w)$. There is another kind of pattern for which this analogy fails:

Definition 5.6. Let $w \in I^{u}$. A real pattern of the second kind for $w$ is a sequence of integers $i<j<g<k$ subject to the following conditions:

1) $i \leq n ; g<i+n ; k<j+n$;

2) $L(w)_{j}>L(w)_{i} \geq L(w)_{k}>L(w)_{g}$.

If $P=(i, j, g, k)$ is such a pattern, $w_{P}$ is obtained from $w$ by $j \downarrow k, i \downarrow j$, and finally, $i \downarrow g$. 


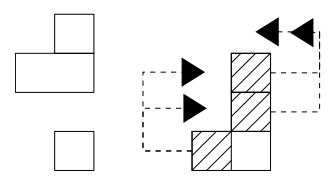

Figure 8. A real pattern of the second kind (left). Here $n=d=4$, and the pattern is $(1,2,3,4) . w_{P}$ is shown in the middle, together with the four up-exchanges defined at $w_{P}\left(\right.$ note that $\operatorname{codim}_{w}\left(w_{P}\right)=3$ ).

Again, $k$ is never equal to $i+n$ in such a pattern. However, the case $k=i+n$ will be what we call an exceptional pattern below. $w_{P}$ is defined by $L\left(w_{P}\right)_{i}=L\left(w_{P}\right)_{g}, L\left(w_{P}\right)_{j}=L\left(w_{P}\right)_{i}$, $L\left(w_{P}\right)_{g}=L\left(w_{P}\right)_{k}$, and $L\left(w_{P}\right)_{k}=L\left(w_{P}\right)_{j}$.

Proposition 5.7. If $P$ is a real pattern of the second kind for $w \in I^{u}$, then $w_{P}$ is singular in $X(w)$. In fact, $\left|E\left(X(w), w_{P}\right)\right|>\operatorname{dim} X(w)$, if $i+n>k$.

Proof. The reasoning is similar to the case of real patterns of the first kind. Again, we first assume that $i+n>k$. Let $w_{1}$ be obtained from $w$ by down-exchanging $j$ and $k$. Then $w>w_{1}>w_{P}$. Let $c_{2}$ be the codimension of $X\left(w_{P}\right)$ in $X\left(w_{1}\right)$. Then there are at least $c_{2}$ up-exchanges $s$ at $w_{P}$ such that $s w_{P} \leq w_{1}$.

Let $c_{1}$ be the codimension of $X\left(w_{1}\right) \subset X(w)$; then there are $c_{1}$ corresponding reflections, all of them involving $j$ or $k$ (and exactly one, both). For each such reflection $s$, let $s^{\prime}$ be the upexchange of $w_{P}$ as follows: if $s$ is $k \uparrow j$, then $s=s^{\prime}$ is defined. Suppose $s$ involves $h \in G_{\geq}(w, j, k)$ : if $s$ up-exchanges $k$ and $h$, then the very same up-exchange is defined at $w_{P}$, and $s^{\prime}=s$ (as then $\left.l_{j}=L\left(w_{P}\right)_{k}>L\left(w_{P}\right)_{h}\right)$. Otherwise, $s$ exchanges $h$ and $j$ (and then $l_{h}>l_{k}$ ); if $l_{h}>l_{i}$, the same up-exchange is defined and again $s=s^{\prime}$. Finally, if $l_{h} \leq l_{i}$, then if $h>g$, replace $s$ by $h \uparrow g$; otherwise, if $h<g$, by $h \uparrow i$. Notice that the case $h=g$ does not occur, because $l_{g}<l_{k}$.

In all these cases, $s^{\prime} w_{P} \leq w$ : If $s^{\prime}$ is $j \uparrow k$, then $s^{\prime} w_{P}$ is obtained from $w$ by $i \downarrow k$ and then $i \downarrow g$. If $s^{\prime}$ involves $k$, but not $j, s^{\prime} w_{P}$ obtained from $w$ by $j \downarrow h, j \downarrow k, i \downarrow j$, and $i \downarrow g$. If $s^{\prime}$ is $h \uparrow j$ for some $h$, then $s^{\prime} w_{P}$ is the result of $j \downarrow h, h \downarrow k, i \downarrow g$, and $i \downarrow h$. If $s^{\prime}$ is $h \uparrow i$, then recall that $h<g$, and $l_{h} \leq l_{i}$; so $s^{\prime} w_{P}$ is obtained from $w$ by $j \downarrow h, i \downarrow j, j \downarrow g$, and $g \downarrow k$. If $s^{\prime}$ is $h \uparrow g$, then $h>g$, and $l_{h} \leq l_{i}$; so $s^{\prime} w_{P}$ is obtained from $w$ by $j \downarrow g, i \downarrow j, g \downarrow h$, and finally, $h \downarrow k$.

Notice that none of the $s^{\prime}$ are among the $c_{2}$ up-exchanges corresponding to $w_{1}>w_{P}$ since $s^{\prime} w_{P} \not \leq w_{1}$ : this is clear if $s^{\prime}$ involves $k$, as $l_{j}=L\left(w_{P}\right)_{k}$ is the largest relevant length. So suppose otherwise; if $s^{\prime}$ involves $h$ and $j$, then $l_{h}>l_{i}$, and so the position of $h_{j}\left(s^{\prime} w_{P}\right)$ in $s w_{P}^{\prime}$ is equal to the position of $h_{h}\left(w_{1}\right)>h_{j}\left(s w_{P}^{\prime}\right)$ in $w_{1}$. If $s^{\prime}$ involves $h$ and $i$, then an easy calculation shows that

$$
\left|\left\{m \in w_{1} \mid m \leq h_{i}\left(s^{\prime} w_{P}\right)\right\}\right|<\left|\left\{m \in s^{\prime} w_{P} \mid m \leq h_{i}\left(s^{\prime} w_{P}\right)\right\}\right| .
$$

Similarly, if $s^{\prime}$ is the up-exchange of $h$ and $g$, then the same is true with $h_{i}\left(s^{\prime} w_{P}\right)$ replaced by $h_{g}\left(s^{\prime} w_{P}\right)$. Thus, we have a combined total of $c_{1}+c_{2}$ up-exchanges. But there is at least one additional up-exchange, namely the one of $k$ and $g$ : Notice that since $g \notin G_{\geq}(w, j, k)$, this one is not among the $c_{1}$ reflections $s^{\prime}$. Moreover, the result is not contained in $w_{1}$ since $L\left(w_{P}\right)_{k}=l_{j}$ is the longest relevant length and would have to be at the same position as in $w_{1}$. All in all, this shows that $\operatorname{dim} E\left(X(w), w_{P}\right)>\operatorname{dim} X(w)$, and we are done.

Now suppose that $k>i+n$. Then $i<[k]$ and $l_{i}>l_{[k]}$, and it is possible, that the up-exchange of $g$ and $k$ in the reasoning above has been listed before. However, we may up-exchange $g$ and $j$ twice, resulting in $w^{\prime} \leq w$, satisfying $L\left(w^{\prime}\right)_{i}=l_{g}, L\left(w^{\prime}\right)_{j}=l_{i}+1, L\left(w^{\prime}\right)_{k}=l_{j}, L\left(w^{\prime}\right)_{g}=l_{k}-1$ (this last value is $\geq 1$ if $g>n$, as then $l_{k}>l_{g} \geq 1$ ). $w^{\prime}$ is obtained from $w$ by down-exchanging $j$ and $i$ (if $l_{j}>l_{i}+1$ ), and then down-exchanging $i$ and $k$, and then $i$ and $g$. As a double upexchange it corresponds to a large reflection, $w_{P}$ is singular. 


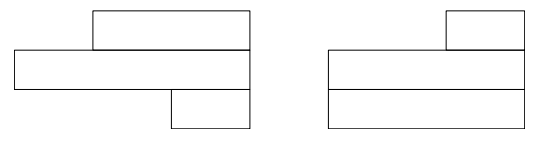

Figure 9. $w$ (left) and $w_{P}$ for the exceptional pattern of the first kind $P=(2,4,5,6)$ (assuming $\left.n=3\right)$.

Remark 5.8. As pointed out by one of the referees, in some instances the fact that $w_{P}$ singular can be seen quicklier. The situation is as follows: Let $L(w)=\left(l_{1}, l_{2}, \ldots, l_{2 n}\right)$ and suppose $P=(i, g, j, k)$ is a real pattern of the first kind, say, where $k<i+n$. For $\sigma \in \mathfrak{S}_{n}$, the symmetric group in $n$ letters, viewed as the permutations of $\{i . i+1, \ldots, i+n\}$, define $\sigma w$ as the element obtained from $w$, by permuting $(i, i+1, \ldots, i+n-1)$ according to $\sigma$. Let $L_{1} \leq L_{2} \leq \cdots \leq L_{k}$ be the distinct values of $l_{i}, l_{i+1}, \ldots, l_{i+n-1}$, and finally put $d_{j}=\left|\left\{i \leq t<i+n \mid \ell_{t}(w)=L_{i}\right\}\right|$. Consider the variety $\mathcal{F}=\mathcal{F}\left(d_{1}, d_{2}, \ldots, d_{k}\right)$ of partial flags $0 \subset V_{1} \subset V_{2} \subset \cdots \subset V_{k} \subset \mathbb{C}^{n}$ with $\operatorname{dim} V_{i}=d_{i} . w$ naturally defines a point in $\mathcal{F}$, which in turn can be described by any element $\tau$ in $\mathfrak{S}_{n}$, for which $\tau(i), \tau(i+1), \ldots, \tau\left(i+d_{1}-1\right)$ are the indices of the basis elements in $V_{1}$ (here, the indices of the $l_{i}$ for which $l_{i}=L_{1}$ ), and so on. If we choose $\tau$ correctly, the existence of the pattern $P$ then means that $\tau$ may be chosen in a way such that $i, g, j, k$ appears in order $k, g$, $j, i$ which is a (Type II) pattern in the classical sense. In particular, $w_{P}$ then corresponds to $\tau_{P}$ obtained from $\tau$ by reordering $k, g, j, i$ to $g, i, k, j$ (and indeed, $w_{P}$ is obtained by replacing $l_{i}$ with $l_{g}, l_{j}$ with $l_{i}, l_{g}$ with $l_{k}$, and $l_{k}$ with $l_{j}$.

Also, if $r$ is a transposition exchanging $p$ and $q$, say, then $\tau_{P}<r \tau_{P} \leq w_{P}$ if and only if $w_{P}<r w_{P} \leq w$ (if we choose the right order on $\mathfrak{S}_{n}$ ). Here $r w_{p}$ means switching $l_{p}$ and $l_{q}$ (corresponding to an up- or down-exchange, or if $l_{p}=l_{q}$ to doing nothing). Since $\tau_{P} \leq \tau$ is a singular point of the corresponding classical Schubert variety, $w_{P}$ is singular in $w$. It is very likely that this can carried over to some of the other patterns. However, the situation is unclear when $i+n<k$ and it seems that we cannot avoid these patterns to find "combinatorial" singularities (i.e. points where we have too many $\widehat{T}$-stable curves); see Remark 6.17 for an example where an exceptional pattern (defined below) is needed.

\subsection{Exceptional patterns}

We conclude this section with two degenerations of real patterns.

Definition 5.9. Let $w \in I^{u}$ and $L(w)=\left(l_{1}, \ldots, l_{2 n}\right)$. Then a sequence $P=(i, g, j=i+n, k)$ with $i<g<j<k$ is called an exceptional pattern of the first kind, if $k<g+n$, and if $l_{i}>l_{[g]}+1$, and $l_{g} \geq l_{k}$. The associated point $w_{P}$ is defined by first down-exchanging $i$ and $g$, and then down-exchanging $g$ and $j$ (only if $l_{i}>l_{g}+1$ ), and finally down-exchanging $g$ and $k$ as before.

Notice that if $g>n$, and $l_{i}=l_{[g]}+1$, the second down-exchange of $i$ and $g$ is void. Notice that the requirement $k<g+n$ is not really necessary. If $k>g+n$, then $g<n$. The above procedure then results in a point $w_{P}<w_{Q}$ for the imaginary pattern $Q=(i, g)$, which clearly is singular. In fact this immediately shows that $w_{P}$ is singular, whenever $g \leq n$. In general, the argument is similar:

Lemma 5.10. Let $P$ be an exceptional pattern of the first kind for $w$. Then $w_{P}$ is a singular point of $X(w)$.

Proof. By the remarks preceding the lemma, we may assume that $g>n$. Then $w_{P}$ is characterized by $L\left(w_{P}\right)_{[g]}=l_{[k]}, L\left(w_{P}\right)_{i}=l_{i}-1, L\left(w_{P}\right)_{[k]}=L(w)_{[g]}+1$. As $[k]>i$, and $l_{i} \geq l_{g}+1=l_{[g]}+2$, it follows that $w_{P}=w_{Q}^{\prime}$ where $w^{\prime}$ is obtained from $w$ by $g \downarrow k$, and $Q$ is the imaginary pattern $(i,[k])$ for $w^{\prime}$. Hence, the claim. 

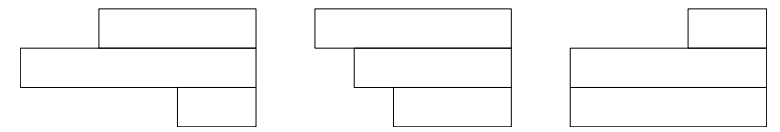

Figure 10. $w$ (left), $w_{P, 1}$, and $w_{P, 2}$ (right) for the exceptional pattern $P=(1,2,3,4)$ of the second kind (assuming again that $n=3$ ). Note that $w_{P, 2}$ equals $w_{Q}$ where $Q=(2,4,5,6)$ is the pattern of the first kind from Fig. 9; it is often the case, that exceptional patterns of the first kind give rise to a pattern of the second kind.

Remark 5.11. It is clear that an exceptional pattern of the first kind is only interesting if $g>n$, and $l_{i}=l_{g}+1$. In all other cases, $w_{P}<w_{Q}$ where $Q=(i, g)$.

As for the second kind, the situation is similar:

Definition 5.12. Let $w \in I^{u}$, then a sequence $P=(i, j, g, k=i+n)$ is called an exceptional pattern of the second kind for $w$ or $L(w)=\left(l_{1}, \ldots, l_{2 n}\right)$, if $i<j<g<k$, and if $l_{i}<l_{j}$, and $l_{i}>l_{g}$.

Then $w_{P, 1}$ is defined as $w_{Q}^{\prime}$ where $w^{\prime}$ is obtained by $i \downarrow j$, and $Q$ is the imaginary pattern $([j],[g])(\operatorname{resp} .([j], g))$ in $w^{\prime}$, if $[j]<[g]$ (resp. $\left.[j]>[g]\right) ; w_{P, 2}$ is defined as $i \downarrow j, i \downarrow j, i \downarrow g$ applied to $w$.

Notice that $Q$ is indeed an imaginary pattern in $w^{\prime}$, because $L\left(w^{\prime}\right)_{i}=l_{j}-1, L\left(w^{\prime}\right)_{j}=l_{i}+1$, $L\left(w^{\prime}\right)_{g}=l_{g}<l_{i}$. Since $w_{Q}^{\prime}$ admits a large reflection relative to $w^{\prime}, w_{Q}^{\prime}$ is singular in $X(w)$.

As for $w_{P, 2}$, it need not always be singular. Indeed, if $l_{j}=l_{i}+1$, then $w_{P, 2}$ is just $i \downarrow g$, $j \downarrow g$ applied to $w$. However, if $l_{i}<l_{j}-1$, then $w_{P, 2}=w_{Q}^{\prime \prime}$ where $w^{\prime \prime}$ is obtained by $i \downarrow g$, and $Q=(j, g)$. Also notice that $w_{P, 2}$ is the point obtained by applying the rule for secondary kind patterns (ignoring that $k=i+n$ ).

Remark 5.13. The exceptional patterns of the second kind are interesting only, if $l_{g}=l_{i}-1$. For suppose $l_{g}<l_{i}-1$. Then, we actually have $w_{P, 1 / 2} \leq w_{Q}$ for $Q=(i, g)$. Also, if $l_{i}<l_{j}-2$, then $w_{P, 2} \leq w_{Q}$ for $Q=(i, j)$.

\section{Two classes of Schubert varieties}

We end this note by studying two classes of affine Schubert varieties where our discussion determines the singular locus completely.

\section{1 $\quad \mathcal{P}$-stable Schubert varieties}

Recall that an affine Schubert variety $X(w)$ is $\mathcal{P}$-stable if and only if $\ell_{1}(w) \geq \ell_{2}(w) \geq \cdots \geq$ $\ell_{n}(w)$ : this is clearly necessary, for in order to be $\mathcal{P}$-stable, it must be $\mathrm{SL}_{n}(K)$-stable, and therefore invariant under the finite Weyl group $W=W_{\mathcal{P}}$; conversely being stable under $W_{\mathcal{P}}$ is clearly enough by the Bruhat decomposition, and it is not hard to see that $X(w)$ is $W$-stable if and only if $W w \subset X(w)$.

In other words, $X(w)$ is $\mathcal{P}$-stable if and only if $\Sigma(w)$ is an actual Young diagram. It is worth mentioning, that $\mathcal{P}$ acts linearly on $V$. In fact, if $\mathcal{P}_{0}=\mathrm{GL}(A)$, then the image of $\mathcal{P}_{0}$ in $\mathrm{GL}(V)$ is precisely $C(u)$, the centralizer of $u$. And, as far as the action on $G(d, V)$ is concerned, $\mathcal{P}_{0}$ and $\mathcal{P}$ have the same orbits. By abuse of language we say $w \in I^{u}$ is $\mathcal{P}$-stable, if $X(w)$ is. Since $e$ is the only $\mathcal{P}$-fixed point in $X\left(w^{s}\right)$, this should not lead to confusion. 

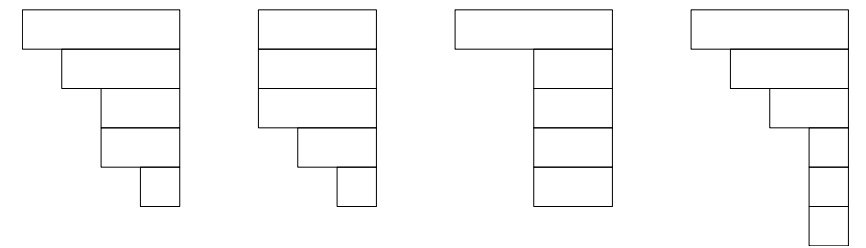

Figure 11. An example of a $\mathcal{P}$-stable $w$ (left), and the three maximal elements of the singular locus of $X(w)$. Here $n=6, s=2$, and the corresponding imaginary patterns are $(1,3),(2,5)$, and $(4,6)$.

For $\mathcal{P}$-stable elements of $I^{u}$, the Bruhat-Chevalley ordering is much simpler: if $x, w \in I^{u}$ are $\mathcal{P}$-stable, then $x \leq w$ if and only if for $k=1,2, \ldots, n$ :

$$
\sum_{i=1}^{k} \ell_{i}(x) \leq \sum_{i=1}^{k} \ell_{i}(w) .
$$

Notice, that if $\Sigma(w)$ is a Young diagram, there are no real patterns. This is clear for those of the first kind. For those of the second, assume $P=(i, j, g, k)$ is such a pattern, then $\left.L(w)_{i}<L_{(} w\right)_{j}$ is possible only if $j>n$. But the requirement that $L(w)_{i} \geq L(w)_{k}>L(w)_{g}$ then implies that $g>k$, a contradiction. But there may be imaginary patterns: let $1 \leq i_{1}<i_{2}<\cdots<i_{k}<n$ be the uniquely determined sequence of those integers satisfying $\ell_{i_{k}+1}(w)<\ell_{i_{k}}(w)$. If $w \neq e$, there is of course at least one such integer. For each $r=1,2, \ldots, k$, let $j_{r}$ be the minimal index, such that $\ell_{j_{r}}(w)<\ell_{i_{r}}(w)-1$. Such an index does not necessarily exist for the last integer $i_{k}$. If it doesn't, we remove $i_{k}$ from the list and replace $k$ with $k-1$. It always exists for $i_{k-1}$. Thus, if $w \neq e$, there is at least one pair $\left(i_{r}, j_{r}\right)$.

Proposition 6.1. Let $w \in I^{u}$ be $\mathcal{P}$-stable. Keeping the notation above, for $1 \leq r \leq k, P_{r}=$ $\left(i_{r}, j_{r}\right)$ is an imaginary pattern of $L(w)$, and the maximal singularities of $X(w)$ are among the points $w_{P_{r}}(1 \leq r \leq k)$. More precisely, $w_{P_{r}}$ is maximal if and only if $i_{r}=\max \left\{i_{h} \mid j_{h}=j_{r}\right\}$.

Proof. It is clearly safe to assume that $w \neq e$. We have noted that $\Sigma(w)$ is a Young diagram. As $X(w)$ is $\mathcal{P}$-stable so is its singular locus. Consequently, if $x$ is a maximal singularity of $X(w)$, then $\Sigma(x)$ is a Young diagram as well. By (9) we have for all $h=1,2, \ldots, n$

$$
\sum_{j=1}^{h} \ell_{j}(x) \leq \sum_{j=1}^{h} \ell_{j}(x) .
$$

Let $i$ be the first index such that $\ell_{i}(x)<\ell_{i}(w)$. Clearly $i$ exists and $\ell_{j}(x)=\ell_{j}(w)$ for $j<i$. Let $i_{0}^{\prime}$ be the maximal row index such that $\ell_{i_{0}^{\prime}}(w)=\ell_{i}(w)$. In other words,

$$
i_{0}^{\prime}=\max \left\{i \leq h \leq n \mid \ell_{h}(w)=\ell_{i}(w)\right\}
$$

Let $j_{0}$ be the minimal row index such that $\ell_{j_{0}}(w) \leq \ell_{i_{0}}(w)-2$. Notice that $j_{0}$ exists if $x$ exists: as $\ell_{i}(x)<\ell_{i}(w)$, there must be a row with index $h>i$ such that $\ell_{h}(x)>\ell_{h}(w)$. As $\ell_{i}(x) \geq \ell_{h}(x)$, it follows $\ell_{i}(w)>\ell_{h}(w)+1$. Finally, let

$$
i_{0}=\max \left\{i^{\prime} \geq i_{0}^{\prime} \mid \ell_{i^{\prime}}(w) \geq \ell_{j_{0}}(w)+2\right\} .
$$

Clearly, $Q=\left(i_{0}, j_{0}\right)$ is among the imaginary patterns constructed above, i.e. $\left(i_{0}, j_{0}\right)=\left(i_{r}, j_{r}\right)$ for some $r$. By construction it is also clear, that $w_{Q}$ is $\mathcal{P}$-stable. Using (10), it follows that $x \leq w_{Q}$, and therefore $x=w_{Q}$. 

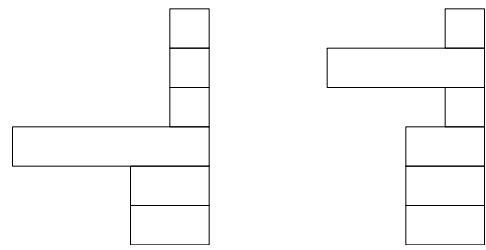

Figure 12. Two elements consisting of one string with critical index $c=3(n=6, s=2) . \kappa^{3}$ is shown on the left.

Remark 6.2. As mentioned, any $\mathcal{P}$-stable $w$ has singularities unless $w=e$ : if $w \neq e$, then $\ell_{1}(w)>\ell_{n}(w)+1$, and therefore $(1, n)$ is always an imaginary pattern for $w$. In addition it follows from the description of the $w_{P_{r}}$ that any $\mathcal{P}$-stable $w^{\prime}<w$ is singular. Consequently, the regular locus of $X(w)$ is just the open orbit $\mathcal{P} w$. This is well known; see [8, Theorem 0.1] for a more general result. It is also shown in [21], where in addition the explicit types of the maximal singularities are described.

In the case $s=1$, (hence $d=n), X\left(w^{s}\right)$ contains the nilpotent cone, i.e. the cone of nilpotent matrices, as an open affine $\widehat{T}$-stable neighborhood $U$ of $e$ (cf. Lusztig's isomorphism). In this setting, for any $\mathcal{P}$-stable $X(w)$, the intersection $X(w) \cap U$ is the closure of a nilpotent orbit in $U$. For these, again, it is well known that they are singular along all smaller orbits ([14]).

\section{2 w consisting of one string}

The second class of Schubert varieties we will be considering now are those, where the "relevant" part of $w$ consists of one string (relevant meaning the part where $w$ differs from $e$ ). In what follows let $e=q_{1}<q_{2}<\cdots$.

Definition 6.3. Let $w \in I^{u}$. Then $w$ consist of one string with critical index $c$ if for all $k>c$ we have $w_{k}=q_{k}=d(n-1)+k$ (and $c$ is minimal with this property), and $w_{1}, w_{2}, \ldots, w_{c}$ are all congruent $\bmod n$, i.e. there is $j$ such that $w_{i} \in S_{j}(w)$ for $1 \leq i \leq c$.

Examples of such $w$ are of course $e$ (with critical index $c=0$ ) and $w^{s}$ (resp. $w_{0}^{s}$ ) (with critical index $c=d$ ). More generally for $c=1, \ldots, d$ let $\kappa^{c}$ be defined as follows

$$
\kappa_{i}^{c}= \begin{cases}(d-c)(n-1)+(i-1) n+1, & i \leq c, \\ d(n-1)+i, & i>c .\end{cases}
$$

Then $\kappa^{d}=w^{s}$. By definition $\kappa^{c}$ consists of one string with critical index $c$. And indeed it is the maximal such element of $I^{u}$. In fact, it is the maximum of all elements $w$ in $I^{u}$ for which $w_{i}=e_{i}$ whenever $i>c$ (that is, $w$ need not consist of one string; see the next lemma).

The main reason, why the $w$ consisting of one string are handled easily, is the following:

Lemma 6.4. Let $w \in I^{u}$ consist of one string with critical index $c$. For any $x \in I^{u}$, we have $x \leq w$ if and only if $x_{c} \geq w_{c}$, and $x_{i}=w_{i}$ for $i>c$.

Proof. We may assume that $c>1$. The only if part being clear, suppose $x_{i}=w_{i}$ for all $i>c$ and $x_{c} \geq w_{c}$. Notice that for $i=0,1, \ldots, c-1$,

$$
w_{c-i}=w_{c}-i n
$$

since $w$ consists of one string. On the other hand, for any $v \in I^{u}$, we always have $v_{i+1}-v_{i} \leq n$, and hence

$$
x_{c-i} \geq x_{c}-i n \geq w_{c}-i n=w_{c-i},
$$

and $x \leq w$ follows. 
We will need the following technical criterion below.

Lemma 6.5. Let $w \in I^{u}$ and suppose $w$ coincides with $e$ at positions $>r$. Then $w$ consists of one string with critical index $c$ if and only if $w_{c-1} \equiv w_{c} \bmod n$ (assuming that $\left.c>1\right)$.

Proof. The only if part is clear. So suppose $w_{c-1} \equiv w_{c} \bmod n$. Then of course $w_{c}=w_{c-1}+n$. For any $0<i<c-1$, there is $h$ such that $w_{c-1}<w_{i}+h n \leq w_{c}=w_{c-1}+n$. But $w_{i}+h n$ belongs to $w$. Hence $w i+h n=w_{c}$.

Since the Bruhat-Chevalley order is that simple for the case of $w$ consisting of one string, the restriction of $s_{\hat{\alpha}}$ being small in Lemma 4.11 is unnecessary.

Lemma 6.6. Let $w$ consist of one string. Keeping the notation of Lemma 4.11, for any $\hat{\alpha}$ such that $\xi_{\hat{\alpha}}$ is defined at $x \leq w$, we have:

$$
s_{\hat{\alpha}} x \leq w \Leftrightarrow r_{i+h+k n, j+k n} x \leq w \text { for all } k \text { such that } 0 \leq k \leq k_{0} .
$$

Proof. Let us denote $r_{i+h+k n, j+k n}$ by $r_{k}$. Clearly $r_{k} x \leq w$ whenever $s_{\hat{\alpha}} x \leq w$ as this direction of the assertion holds for any $w\left(r_{k} x \leq s_{\hat{\alpha}} x\right.$ if $\left.s_{\hat{\alpha}} x>x\right)$; thus, we may assume $r_{k} x \leq w$ for all $k$, and it is also safe to assume that $s_{\hat{\alpha}} x>x$. Let $c>0$ be the critical index of $w$. By Lemma 6.4 we have to show that $\left(s_{\hat{\alpha}} x\right)_{l} \geq w_{l}$ for $l \geq c$.

As $r_{k} x \leq w$, it is clear that $\left(r_{k} x\right)_{l}=x_{l}=w_{l}$ for $l>c$, which easily implies that $r_{k}\left(x_{l}\right)=x_{l}$ and $\left(s_{\hat{\alpha}} x\right)_{l}=w_{l}$ for $l>r$. Thus, the only problem might arise if $\left(s_{\hat{\alpha}} x\right)_{c} \neq x_{c}$. In this case, there is some $k$ such that $\left(r_{k} x\right)_{c}<x_{c}$. Thus, $r_{k}$ moves $x_{c}$. It follows that $k=r_{0}$. Then, if $\left(r_{k} x\right)_{c}=r_{k}\left(x_{c}\right)$ we are done, for in this case $\left(s_{\hat{\alpha}} x\right)_{c}=r_{k}\left(x_{c}\right) \geq w_{c}$ by assumption. The remaining case is $\left(r_{k} x\right)_{c}=x_{c-1}$ (equivalent to $\left.r_{k}\left(x_{c}\right)<x_{c-1}\right)$. Now, if $x_{c-1}$ and $x_{c}$ are not congruent mod $n$, we are again done, for then $s_{\hat{\alpha}}\left(x_{c-1}\right)=x_{c-1}=\left(s_{\hat{\alpha}} x\right)_{c} \geq w_{c}$ because $r_{k} x \leq w$.

Finally, if $x_{c-1}$ and $x_{c}$ are congruent $\bmod n$, then $x$ itself consists of one string by Lemma 6.5. But if $x$ itself consists of one string, then $\left(s_{\hat{\alpha}} x\right)_{c}=s_{\hat{\alpha}}\left(x_{c}\right)=r_{k}\left(x_{c}\right)$. If $r_{k}\left(x_{c}\right)<w_{c}$, then $\left(r_{k} x\right)_{c}=x_{c-1}=x_{c}-n<w_{c}-$ a contradiction $\left(w_{c}+n\right.$ occurs in both, $|x|$ and $|w|$ at positions strictly bigger than $c)$. Hence, $\left(s_{\hat{\alpha}} x\right)_{c}=r_{k}\left(x_{c}\right) \geq w_{c}$.

Corollary 6.7. Suppose $\xi_{\hat{\alpha}}$ is defined at $x \leq w \in I^{u}$ where $w$ consists of one string. Then $\xi_{\hat{\alpha}} \in T_{x}(X(w))$ if and only if $s_{\hat{\alpha}} x \leq w$. In particular,

$$
T_{x}(X(w))_{\mathrm{re}}=T E(X(w), x) .
$$

Proof. Suppose $\hat{\alpha}<0$. Then $s_{\hat{\alpha}} x>x$ by Lemma 4.8. If $\xi_{\hat{\alpha}} \in T_{x}(X(w))$, then $\xi_{\hat{\alpha}} \in T_{x}(Y(w))$, and therefore (using the notation of Lemma 4.11 and the proof of Lemma 6.6), $r_{k} x \leq w$ for all $0 \leq k \leq k_{0}$. Lemma 6.6 now gives $s_{\hat{\alpha}} x \leq w$. All other cases are immediate.

In the case of imaginary tangents, a similar result holds:

Lemma 6.8. Let $w \in I^{u}$ consist of one string with critical index $c$. If for any $x \leq w, S(h, x, w)$ (cf. (8)) contains $i \neq j$, say, then $\xi_{(i j), h} \in T_{x}(X(w))$. In particular, $x$ is singular.

Proof. Without loss of generality, $h_{i}(x)<h_{j}(x)$. Let $m$ be the unique nonnegative integer such that $h_{j}(x)-h_{i}(x)=(j-i)+m n$. Let $\hat{\beta}=(i j)-m \delta$. Then $\hat{\beta}<0$ by construction. Moreover, $s_{\hat{\beta}} x=x$ because $s_{\hat{\beta}}\left(h_{j}(x)\right)=h_{i}(x)$. Furthermore, $s_{\hat{\beta}-h \delta} x \leq w$. To see this, notice that as $S(x, h, w)$ contains two or more elements, $x$ cannot consist of one string with critical index greater or equal $c$. It is clear that $s_{\hat{\beta}-h \delta} x \in I^{u}$ (because $\ell_{j}(x) \geq h$ and $h_{i}(x)-h n>0$ ), and is obtained from $x$ by increasing $\ell_{i}(x)$ and decreasing $\ell_{j}(x)$ by $h$. By hypothesis, $w$ consists of one string, so we have to see that $\left(s_{\hat{\beta}-h \delta} x\right)_{k}=w_{k}$ for $k>c$, and $\left(s_{\hat{\beta}-h \delta} x\right)_{c} \geq w_{c}$. The first assertion is clear, because $s_{\hat{\beta}-h \delta}$ changes only those elements of $x$ which are also changed by one 

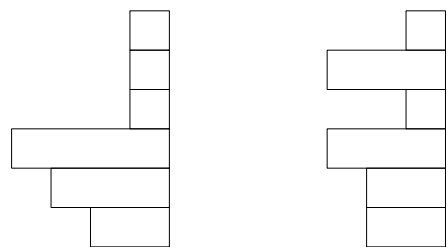

Figure 13. $\varphi(w)$ for the elements consisting of one string shown in Fig. 12. $\varphi\left(\kappa^{3}\right)$ is left.

of the $r_{h_{i}(x)+(k-h) n, h_{i}(x)+k n}$ or $r_{h_{j}(x)+(k-h) n, h_{j}(x)+k n}$, and these do not change the entries $x_{l}$ for $l>c$.

There are two possibilities: Either $s_{\hat{\beta}-h \delta}$ does not change $x_{c}$ and we are done, or $\left(s_{\hat{\beta}-h \delta} x\right)_{c}$ is $x_{c-1}$ with $x_{c-1} \notin S_{j}(x): s_{\hat{\beta}-h \delta}\left(x_{c}\right) \neq x_{c}$, therefore $x_{c} \in S_{j}(x)$ is the largest element changed, i.e. $x_{c}=h_{j}(x)+(h-1) n$. As $x$ does not consist of one string with critical index $c, x_{c-1} \notin S_{j}(x)$ (Lemma 6.5), and $x_{c-1}=\left(r_{h_{j}(x)-n, h_{j}(x)+(h-1) n} x\right)_{c}\left(\right.$ recall that $\left.x_{c-1}>x_{c}-n\right)$. As $j \in S(h, x, w)$, this last statement means $x_{c-1} \geq w_{c}$ and so $s_{\hat{\beta}-h \delta} x \leq w$.

But now we conclude, as in the proof of Lemma 4.18 that $\xi_{(i j), h}= \pm\left[\xi_{-\hat{\beta}}, \xi_{\hat{\beta}-h \delta}\right]$ is tangent to $X(w)$ at $x$.

Summarizing, we have obtained:

Theorem 6.9. Suppose $w \in I^{u}$ consists of one string. Then for all $x \leq w$ we have $T_{x}(X(w))=$ $T_{x}\left(X\left(w^{s}\right)\right) \cap T_{x}(Y(w))$. In particular, in $T_{x}(Y(w))^{u}, T_{x}(X(w))$ is given by the trace relations.

Proof. Lemma 6.6 in particular says that if $\xi_{\hat{\alpha}}$ is defined at $x$ and is contained in $T_{x}(Y(w))$, then it is contained in $T_{x}(X(w))$. By Lemma 4.8, this means that

$$
T_{x}(X(w))_{\mathrm{re}}=T_{x}(Y(w))_{\mathrm{re}}^{u}=T_{x}(Y(w)) \cap T_{x}\left(X\left(w^{s}\right)\right)_{\mathrm{re}} .
$$

Regarding imaginary roots, Lemma 6.8 immediately implies that the imaginary part of $T_{x}\left(X\left(w^{s}\right)\right)$ is spanned by all $\xi_{(i j), h}$ for which $i \neq j \in S(h, x)$. In other words, $T_{x}\left(X\left(w^{s}\right)\right)_{-h \delta}$, as a subspace of $T_{x}(G(d, V))_{-h \delta}$, is cut out by the trace relation for $h$ (cf. Lemma 4.14). Applying Lemma 6.8 in the case of arbitrary $w \in I^{u}$, this, combined with Lemma 4.15, means that for each $h, \xi_{(i j), h} \in T_{x}(X(w))$ if and only if $\xi_{(i j), h} \in T_{x}\left(X\left(w^{s}\right)\right)_{-h \delta} \cap T_{x}(Y(w))$. This completes the proof.

Remark 6.10. Theorem 6.9 together with Corollary 6.7 immediately imply that for $w$ consisting of one string, $X(w)$ is smooth at $x \leq w$ if and only if the number of $\widehat{T}$-stable curves in $E(X(w), x)$ equals the dimension of $X(w)$, and if there is no imaginary tangent in $T_{x}(X(w))$. This is consistent (though stronger) with the observation in Remark 4.19, which on the other hand applies to arbitrary $w$ not just those consisting of one string.

Let $w$ consist of one string with critical index $c>2$. If $w=\kappa^{c}$ let $P=\left(\left[w_{1}\right],\left[w_{1}+1\right]\right)$, otherwise put $P=\left(\left[w_{1}\right],\left[w_{c+1}-n\right]\right)$, where we put $w_{c+1}=d(n-1)+c+1$ in case $c=d$. Clearly $P$ is an imaginary pattern for $w$, and we define $\varphi(w)=w_{P}$.

Thus, if $w \neq \kappa^{r}, \varphi(w)$ is obtained from $w$ by replacing $w_{1}$ with $w_{c+1}-n$; and $\varphi\left(\kappa^{c}\right)$ is obtained by replacing $\kappa_{1}^{c}$ with $\kappa_{c}^{r}+1$.

It is clear that $\varphi(w)$ is singular. But in fact we have

Theorem 6.11. Let $w$ consist of one string with critical index $c>2$. Then the singular locus of $X(w)$ is $X(\varphi(w))$.

In particular, the singular locus of $X(w)$ has exactly codimension two.

For the proof we will need: 
Theorem 6.12. Let $x \leq \kappa^{c}$ for some $c>0$. Then the set

$$
E\left(\kappa^{c}, x\right)=\left\{s \in \widehat{W} \mid x \neq s x \leq \kappa^{c}\right\}
$$

has precisely $c(n-1)=\operatorname{dim} X\left(\kappa^{c}\right)$ elements. In particular $\operatorname{dim} T_{x}\left(X\left(\kappa^{c}\right)\right)_{\mathrm{re}}=\operatorname{dim} T E\left(X\left(\kappa^{c}\right), x\right)$ $=\operatorname{dim} X\left(\kappa^{c}\right)$.

Proof. The assertions on the dimension of $T_{x}\left(X\left(\kappa^{c}\right)\right)$ and $T E\left(X\left(\kappa^{c}\right), x\right)$ are immediate consequences of the first assertion and Corollary 6.7.

We proceed by descending induction on $c$. Suppose $c=d$. Then $\kappa^{c}=w^{s}$, and we simply have to count all reflections $s_{\hat{\alpha}}$, defined at $x$, with $s_{\hat{\alpha}} x \neq x$. It will be convenient to use the notation introduced in the beginning: we write $x=\tau^{\left(c_{1}, c_{2}, \ldots, c_{n}\right)}$, then $h_{i}(x)=i+c_{i} n$. We have to count the defined $\xi_{\hat{\alpha}} \mathrm{s}$. $\xi_{\hat{\alpha}}$ may map a given $e_{h_{i}(x)}$ to any $e_{j}$, provided $j \not \equiv i \bmod n, j \notin|x|$, and $h_{[j]}(x)-j \leq n \ell_{i}(x)$ (the latter because $\xi_{\hat{\alpha}}$ is $\tau$-equivariant). By construction it is clear that also $h_{[j]}(x)=[j]+c_{[j]} n$ thus there are at most $c_{[j]}$ such maps sending $e_{h_{i}(x)}$ to an element congruent to $[j]$.

If $E=\left|E\left(\kappa^{c}, x\right)\right|$ we conclude

$$
E=\sum_{i=1}^{n} \sum_{\substack{1 \leq j \leq n \\ j \neq i}} \min \left\{c_{j}, \ell_{i}(x)\right\} .
$$

Of course, $d=\sum_{i} \ell_{i}(x)$, and $c_{j}=d-\ell_{j}(x)=\sum_{i \neq j} \ell_{i}(x)$. Thus, $c_{j} \geq \ell_{i}(x)$ for all $i \neq j$, which implies that

$$
E=\sum_{i} \sum_{j \neq i} \ell_{i}(x)=d(n-1)
$$

This settles the case $c=d$ : we already know $T E\left(X\left(w^{s}\right), x\right)$ contains all $\xi_{\hat{\alpha}}$ that are defined (cf. Lemma 4.8). We find that $\operatorname{dim} T E\left(X\left(w^{s}\right), x\right)=d(n-1)$ for all $x \leq w^{s}$. This applies in particular to $x=w^{s}$ showing that $\operatorname{dim} X\left(w^{s}\right)=d(n-1)$.

Now suppose $c<d$ and the assertion holds for $c^{\prime}>c$. The reflections which we have to count are precisely those which fix $\kappa_{c+1}^{c}, \kappa_{c+2}^{c}, \ldots, \kappa_{d}^{c}: s_{\hat{\alpha}} x \leq \kappa^{c}$ if and only if

$$
\left(s_{\hat{\alpha}} x\right)_{k}=\kappa_{k}^{c}
$$

for $k>c$ and $\left(s_{\hat{\alpha}} x\right)_{c} \geq \kappa_{c}^{c}$. This last condition is void because $\kappa_{c}^{c}=\kappa_{c+1}^{c}-n$.

By induction we may assume that the number of reflections for which (11) holds for $k>c+1$ equals $\operatorname{dim} X\left(\kappa^{c+1}\right)=(c+1)(n-1)$ (note that $\kappa_{k}^{c}=\kappa_{k}^{c+1}$ for these $k$ ). Let $E^{\prime}$ be the set of these reflections. For each $i \in\{1,2, \ldots, n\}$ which is not congruent $\kappa_{c+1}^{c} \bmod n$, there is exactly one reflection $s$ in $E^{\prime}$ for which $(s x)_{c+1} \neq x_{c+1}=\kappa_{c+1}^{c}$ : namely, the one moving the entire $n$-string in $x$ through $x_{c+1}$ to the string through $i$. To be precise, if the congruence class of $x_{c+1}$ is $j$, then $s$ replaces $x$ by removing all elements in $S_{j}(x)$ up until (including) $x_{c+1}$ and replacing them by adding the same number of elements to the string through $i$. This is always possible (as $\ell_{j}(x) \leq c_{i}$ ). Of course, $s$ is an up-exchange, because $x_{c+1}$ is moved to a smaller number. Also, $(s x)_{c+1}<x_{c+1}$, and therefore $s x \not \leq \kappa^{c}$.

Thus $E=c(n-1)$. As $x$ was chosen arbitrary, this in particular says that $E\left(\kappa^{c}, \kappa^{c}\right)$ has $c(n-1)$ elements. $\kappa^{c}$ is a smooth point of $X\left(\kappa^{c}\right)$, so here we know that the number of curves is equal to the dimension of $X\left(\kappa^{c}\right)$, and therefore $\operatorname{dim} X\left(\kappa^{c}\right)=c(n-1)$.

Remark 6.13. An immediate consequence of this theorem is the fact that $X\left(\kappa^{c}\right)$ is rationally smooth (cf. [5]), which of course is well known, at least in the case of $c=d$, and also shown in $[2]$. 
Furthermore, as is easily seen, $T_{e}(X(w))_{\text {re }}=T_{e}\left(X\left(\kappa^{c}\right)\right)_{\text {re }}$ whenever $w$ consists of one string with critical index $c \geq 2$. Therefore, for such a $w$ different from $\kappa^{c}, X(w)$ cannot be globally rationally smooth, as $\operatorname{dim} X(w)<\operatorname{dim} X\left(\kappa^{c}\right)$.

Returning to the situation of an arbitrary $w$ consisting of one string, we have:

Lemma 6.14. Suppose $x \leq w$ both consist of one string with critical index $c>0$. Then $x$ is a regular point of $X(w)$.

Proof. Let $E=\left\{s_{\hat{\alpha}} \mid x \neq s_{\hat{\alpha}} x \leq \kappa^{c}\right\}$. By Theorem 6.12, we know that $|E|=c(n-1)$. Furthermore the codimension $c$ of $X(w)$ in $X\left(\kappa^{r}\right)$ is precisely $w_{1}-\kappa_{1}^{c}=w_{c}-\kappa_{c}^{c}$ (by Lemma 3.8). Now $x$ consists of one string with critical index $c$, and therefore any $s_{\hat{\alpha}} \in E$ satisfies $s_{\hat{\alpha}} x \leq w$ if and only if $s_{\hat{\alpha}}\left(x_{c}\right)=\left(s_{\hat{\alpha}} x\right)_{c} \geq w_{c}$. There are precisely $c$ elements $s_{\hat{\alpha}}$ of $E$ such that $\kappa_{c}^{c} \geq$ $s_{\hat{\alpha}}\left(x_{c}\right)>w_{c}$ : if $s_{\hat{\alpha}} x>x$ and $s_{\hat{\alpha}} \in E$, then $s_{\hat{\alpha}} x$ consists of one string, so $s_{\hat{\alpha}} x$ is uniquely determined by $s_{\hat{\alpha}}\left(x_{c}\right)$. As a consequence $|E(X(w), x)|=\operatorname{dim} X\left(\kappa^{c}\right)-c=\operatorname{dim} X(w)$.

It remains to show that $T_{x}(X(w))$ has no imaginary weight. However, as $T_{x}(X(w)) \subset$ $T_{x}\left(X\left(\kappa^{c}\right)\right)$ this may be checked in case $w=\kappa^{c}$. So suppose $\xi_{i, h}$ is defined at $x$ for some $i, h$, and contained in $T_{x}\left(Y\left(\kappa^{c}\right)\right)$. Then $r_{h_{i}(x)-h n, h_{i}(x)} x \leq \kappa^{c}$. In particular, $h_{i}(x)$ must appear before the critical index $c$ of $x$ and thus $h_{i}(x)=x_{1}$; consequently, no other $\xi_{j, h}$ is contained in $T_{x}\left(Y\left(\kappa^{c}\right)\right)$ for this given $h$. The trace condition now kills $\xi_{i, h}$ (in fact, as $h_{i}(x)=x_{1}$, and $x_{1}-\kappa_{1}^{c}<n$, even $\xi_{i, h}$ is not tangent to $T_{x}\left(Y\left(\kappa^{c}\right)\right)$.

Remark 6.15. Notice that this already shows the (elementary) fact that $X(w)$ is globally nonsingular for all $w$ consisting of one string with critical index $c=1$.

Proof of Theorem 6.11. We have to show that any $x \leq w$ which is not below $\varphi(w)$ is a regular point of $X(w)$. By Lemma 6.14 it suffices to treat the case when $x$ does not consist of one string with critical index $c$ itself.

Let $l=\left|\left\{x_{i} \mid w_{c+1}-n \leq x_{i}<w_{c+1}\right\}\right|$ be the number of entries of $x$ between $w_{c+1}-n$ and $w_{c+1}$. If $l=1$, then $x=\kappa^{c-1}$ since we excluded the remaining possibility that $x$ consists of one string with critical index $c$.

Suppose first that $x_{1} \geq w_{2}$. Then $x_{2} \geq w_{3}, \ldots, x_{c-2} \geq w_{c-1}$. Otherwise $x_{c-2}<w_{c-1}$ (as all $w_{i}$ lie on one string), and thus $x_{h}=x_{c-1}$ is the only entry of $x$ with $w_{c-1} \leq x_{h}<w_{c}$. Consequently the entries $x_{1}, x_{2}, \ldots, x_{c-1}$ are all congruent $\bmod n$, and therefore $x_{1}<w_{c-1}-$ $(c-3) n=w_{2}$, a contradiction. But now $x \leq \varphi(w)$, since $x_{c-1} \geq w_{c+1}-n$ : If $x_{c-1}<w_{c+1}-n$, then $x_{c}=x_{c-1}+n<w_{c+1}$, and thus $l=1$, and therefore $x=\kappa^{c-1}$. Again, this contradicts $x_{1} \geq w_{2}$. Summarizing, if $x_{1} \geq w_{2}$ then $x \leq \varphi(w)$ (obviously this is only-if as well).

It remains to treat the case when $x_{1}<w_{2}$. In this case $l=2$, or $x=\kappa^{c-1}$. In the second case, the only reflections $s$ with $\kappa^{c-1}<s \kappa^{c-1} \leq w$ are precisely the up-exchanges of $i=\left[\kappa_{1}^{c-1}\right]$ with a number between $i$ and $\left[w_{1}\right]$, giving a total of $\kappa_{1}^{c-1}-w_{1}$ which is the codimension of $x=\kappa^{c-1}$ in $X(w)$. In the other case $(l=2)$, we have $x_{1} \equiv x_{2} \equiv \cdots \equiv x_{c-1} \bmod n$ but $x_{1} \not \equiv x_{c} \bmod n$. Thus, the only possibilities for a reflection are to move $x_{c-1}$ to any integer between $w_{c+1}-n$ and $x_{c-1}$, or $x_{c}$ to an integer between $w_{c}$ and $x_{c}$, or finally, $x_{c-1}$ to $x_{c}-n$ (one might think one could move $x_{c-1}$ also to values between $w_{c-1}$ and $w_{c+1}-n$, but that is not possible in general since such a reflection would have to move $x_{c-1}+n \geq w_{c+1}$ as well. Counting these reflections gives $\left(x_{c-1}-w_{c+1}-n\right)+\left(x_{c}-w_{c}\right)+1$. But this is just the codimension of $X(x)$ in $X(w)$ ( $x$ is obtained from $w$ by first down-exchanging $\left[w_{1}\right]$ and $\left[x_{1}\right]$, and then down-exchanging $\left[w_{c}\right]$ and $\left.\left[x_{c}\right]\right)$. Concluding it follows that $E(X(w), x)$ has the minimal number of elements possible, namely $\operatorname{dim} X(w)$. On the other hand, there is no imaginary tangent at $x$ : if $l=1$ the only possibility for such a tangent is $\xi_{\left[x_{1}\right], 1}$ but $x_{1}-n<w_{1}$. If $l=2$ there is at most one other possibility (corresponding to $\left[x_{c}\right]$ ) but this is killed by the trace relation (or the remark that $x_{c}-n<w_{c}$ and $\left.x_{c-1}<w_{c}\right)$. Theorem 6.9 now gives the result. 
Finally, that $X(\varphi(w))$ has codimension two in $X(w)$ follows from the fact that here $\varphi(w)=$ stw where $s, t$ are small reflections and $s t w<t w<w$ are codimension one steps (cf. Lemma 3.8).

Remark 6.16. In view of Remark 6.15 the only remaining case is $w$ consisting of one string with critical index $c=2$. The only difference to the case $c>2$ is that $\varphi(w)$ has to be defined slightly different: the pattern for such a $w$ is $P=\left(\left[w_{1}\right],\left[w_{1}\right]+1\right)$ (notice that $\left.\left[w_{1}\right]=\left[w_{2}\right]\right)$. All the proofs above go through when this is kept in mind appropriately. The main difference now is that $X(\varphi(w))$ does not have codimension two as in the case of critical index $c>2$.

Remark 6.17. Finally, let us conclude with a short remark on the smooth Schubert varieties: In [2] Billey and Mitchell completely classify all smooth Schubert varieties in affine Grassmannians for all types. They all are (closed) orbits for certain parabolic ind-subgroups of $\mathcal{G}$. In type $A$, their result means (in our notation) that the smooth $X(w)$ are precisely those where $L(w)=\left(l_{1}, l_{2}, \ldots, l_{2 n}\right)$ has the following form (we list only $\left.l_{1}, l_{2}, \ldots, l_{n}\right)$ : There is a pair of integers $1 \leq p<q \leq n-p$ such that $l_{1}=l_{2}=\cdots=l_{p}=1$ and $l_{q}=l_{q+1}=\cdots=l_{q+p}=2$ and $l_{i}=1$ for all remaining $i$ between $p+1$ and $n$. Alternatively (somewhat dual to this construction) there is a second "family" given by integers $1 \leq p<q \leq n-p$ with $l_{n-1}=l_{n-2}=\cdots=l_{n-p}=2$ and $l_{n-q}=l_{n-q-1}=\cdots=l_{n-q-p}=0$. In particular, all of them are below $w^{1}$ (i.e. $s=1$ and $d=n$ ).

This is consistent with our discussion: if $X(w)$ is smooth it cannot admit any imaginary pattern and hence $l_{i}-l_{j}$ cannot be strictly greater than 1 if $i<j$ and 2 if $i>j$. This already shows that $w \leq w^{1}$, and hence $l_{i} \in\{0,1,2\}$ for $i \leq n$. Not allowing any real pattern then asserts that we are in one of the two cases listed. Indeed, it follows that all the $i$ with $l_{i}=2$ are strictly larger than all the $i$ with $l_{i}=0$. Also. there cannot be any "gap" between $i$ and $j>i$ for which $l_{i}=l_{j}=2$; similarly, all the $l_{i}$ with $l_{i}=0$ must be consecutive as well. Assuming $w \neq e$, if $l_{1} \neq 0$, then $l_{n}=2$ for otherwise there is a real pattern $(i, n, n+1, j)$ where $[j]<i$ and $l_{j}=1$.

It seems plausible that one could show the smoothness of these Schubert varieties also from our discussion (maybe using Remark 4.19) by arguing that the classical Schubert varieties $Y(w)$ won't contain any imaginary tangents for all these varieties.

In [2] the rationally smooth Schubert varieties are also classified. There are two types (other than the smooth ones): what the authors call "spiral" corresponds to our $\kappa^{c}$ (and a dual version, related by an automorphism) and we showed above that this is indeed rationally smooth. The second type is "chains", that is, Schubert varieties, that contain exactly one flag of subvarieties $X(e) \subsetneq X\left(w_{1}\right) \subsetneq X\left(w_{2}\right) \subsetneq \cdots \subsetneq X\left(w_{n}\right)=X(w)$ where $n=\operatorname{dim} X(w)$. According to [2], except for the case $n=1$, these are all smooth.

Hence the only rationally smooth Schubert varieties are the smooth ones and (essentially) those of the form $X\left(\kappa^{c}\right)$. As one referee suggested, it is tempting to conjecture that this means all $w$ except $w=\kappa^{c}$ or those for which $X(w)$ is smooth, should admit a real pattern of some sort. While this may be true, consider the following example: $n=d=4$, so $s=1$, and $w=s_{(12)} w^{s}$. Then $w$ consists of one string with critical index $d$. It is not rationally smooth since it is strictly smaller than $\kappa^{d}=w^{s}$. However the only real pattern supported by $w$ is degenerate: it is an exceptional real pattern of the first kind, namely $P=(2,5,6,7)(L(w)=(0,4,0,0,1,5,1,1))$. The corresponding singular point $w_{P}$ is defined as $L\left(w_{P}\right)=(0,3,1,0,1,4,2,1)$. Note that $w_{P}<\varphi(w)$ and indeed, $w_{P}$ admits four up-exchanges whereas the codimension of $X\left(w_{P}\right)$ in $X(w)$ is only three. $\varphi(w)$ on the other hand is still a rationally smooth point of $X(w)$.

\section{Acknowledgments}

The first author was supported by the Swiss National Science Foundation, and partially by an NSERC Discovery Grant. The second author was supported by NSF grant DMS-0652386 and Northeastern University RSDF 07-08. The first author would like to thank the Swiss National 
Science Foundation for making possible his stay at Northeastern University, during which most of this work has been done. We would like to thank the referees for their careful reading and their many valuable suggestions.

\section{References}

[1] Billey S., Braden T., Lower bounds for Kazhdan-Lusztig polynomials from patterns, Transform. Groups 8 (2003), 321-332, math.RT/0202252.

[2] Billey S., Mitchell S., Smooth and palindromic Schubert varieties in affine Grassmannians, arXiv:0712.2871.

[3] Billey S., Postnikov A., Smoothness of Schubert varieties via patterns in root subsystems, Adv. in Appl. Math. 34 (2005), 447-466, math.CO/0205179.

[4] Billey S., Warrington G., Maximal singular loci of Schubert varieties in SL(n)/B, Trans. Amer. Math. Soc. 355 (2003), 3915-3945, math.AG/0102168.

[5] Carrell J., The Bruhat graph of a Coxeter group, a conjecture of Deodhar, and rational smoothness of Schubert varieties, in Algebraic Groups and Their Generalizations: Classical Methods (University Park, PA, 1991), Proc. Sympos. Pure Math., Vol. 56, Part 1, Amer. Math. Soc., Providence, RI, 1994, 53-61.

[6] Carrell J., Kuttler J., Smooth points of $T$-stable varieties in $G / B$ and the Peterson map, Invent. Math. 151 (2003), 353-379, math.AG/0005025.

[7] Cortez A., Singularités génériques et quasi-résolutions des variétés de Schubert pour le groupe linéaire, Adv. Math. 178 (2003), 396-445, math.AG/0106130.

[8] Evens S., Mircović I., Characteristic cycles for the loop Grassmannian and nilpotent orbis, Duke Math. J. 97 (1999), 109-126.

[9] Gasharov V., Sufficiency of Lakshmibai-Sandhya singularity conditions for Schubert varieties, Compositio Math. 126 (2001), 47-56.

[10] Juteau D., Modular representations of reductive groups and geometry of affine Grassmannians, arXiv:0804.2041.

[11] Kassel C., Lascoux A., Reutenauer C., The singular locus of a Schubert variety, J. Algebra 269 (2003), $74-108$.

[12] Kumar S., The nil Hecke ring and singularity of Schubert varieties, Invent. Math. 123 (1996), 471-506, alg-geom/9503015.

[13] Kumar S., Kac-Moody groups, their flag varieties and representation theory, Progress in Mathematics, Vol. 204, Birkhäuser Boston, Boston, MA, 2002.

[14] Kraft H., Procesi C., Minimal singularities in $\mathrm{GL}_{n}$, Invent. Math. 62 (1981), 503-515.

[15] Lakshmibai V., Sandhya B., Criterion for smoothness of Schubert varieties in SL(n)/B, Proc. Indian Acad. Sci. Math. Sci. 100 (1990), 45-52.

[16] Lakshmibai V., Seshadri C.S., Singular locus of a Schubert variety, Bull. Amer. Math. Soc. (N.S.) 11 (1984), 363-366.

[17] Lakshmibai V., Weyman J., Multiplicities of points on a Schubert variety in a minuscule G/P, C. R. Acad. Sci. Paris Sér. I Math. 307 (1988), 993-996.

[18] Lusztig G., Green polynomials and singularities of unipotent classes, Adv. in Math. 42 (1981), $169-178$.

[19] Lusztig G., Canonical bases arising from quantized universal enveloping algebras, J. Amer. Math. Soc. 3 (1990), 447-498.

[20] Magyar P.M., Affine Schubert varieties and circular complexes, math.AG/0210151.

[21] Malkin A., Ostrik V., Vybornov M., Minimal degeneration singularities in the affine Grassmannians, Duke Math. J. 126 (2005), 233-249, math.AG/0305095.

[22] Manivel L., Le lieu singulier des variétés de Schubert, Internat. Math. Res. Notices 2001 (2001), no. 16, 849-871, math.AG/0102124. 